\title{
Influence of ultrasound speckle tracking strategies for motion and strain estimation
}

\author{
Ariel H. Curiale ， Gonzalo Vegas-Sánchez-Ferrero ，Santiago Aja-Fernández'
}

\begin{abstract}
A B S T R A C T
Speckle Tracking is one of the most prominent techniques used to estimate the regional movement of the heart based on ultrasound acquisitions. Many different approaches have been proposed, proving their suitability to obtain quantitative and qualitative information regarding myocardial deformation, motion and function assessment. New proposals to improve the basic algorithm usually focus on one of these three steps: (1) the similarity measure between images and the speckle model; (2) the transformation model, i.e. the type of motion considered between images; (3) the optimization strategies, such as the use of different optimization techniques in the transformation step or the inclusion of structural information. While many contributions have shown their good performance independently, it is not always clear how they perform when integrated in a whole pipeline. Every step will have a degree of influence over the following and hence over the final result. Thus, a Speckle Tracking pipeline must be analyzed as a whole when developing novel methods, since improvements in a particular step might be undermined by the choices taken in further steps. This work presents two main contributions: (1) We provide a complete analysis of the influence of the different steps in a Speckle Tracking pipeline over the motion and strain estimation accuracy. (2) The study proposes a methodology for the analysis of Speckle Tracking systems specifically designed to provide an easy and systematic way to include other strategies. We close the analysis with some conclusions and recommendations that can be used as an orientation of the degree of influence of the models for speckle, the transformation models, interpolation schemes and optimization strategies over the estimation of motion features. They can be further use to evaluate and design new strategy into a Speckle Tracking system.
\end{abstract}

\section{Introduction}

The analysis of the regional motion of the heart has proved to be of preeminent importance for the study of cardiac abnormal behavior. It currently plays an inarguable role in treatment and diagnosis of several pathologies, such as mitral regurgitation (Helmcke et al., 1987; Bargiggia et al., 1991), ischemia (Voigt et al., 2003), dyssynchrony (Suffoletto et al., 2006), myocardial quantification (McDicken et al., 1992; Amundsen et al., 2006; Nesser et al., 2009; Geyer et al., 2010) and diastolic dysfunction (Ommen et al., 2000). The estimation of the features that model regional motion can be done using different imaging modalities, dominant among them those based on ultrasound (US) acquisitions. Due to low cost and real time acquisition, echocardiography has become a widely used tool for motion and strain estimation, either using Doppler or non-Doppler techniques.

Despite its undeniable potential in many cardiac applications, Doppler techniques are limited by inaccuracies due to aliasing, frequency-dependent attenuation and, most importantly, the angle dependence in the assessment of tissue velocities. These limitations have prevented Doppler techniques from becoming a standard in daily praxis (Dandel et al., 2009). In contrast, non-Doppler techniques, generally known as Speckle Tracking, can estimate tissue velocities in the entire image while not suffering from aliasing and being angle independent (Trahey et al., 1988; 1987). 
The term Speckle Tracking (ST) refers to all those techniques that analyze motion by tracking the intensity or the interference patterns of the US data, known as speckle, along the temporal sequences. In the particular case when the motion is analyzed by tracking the speckle from the RF signal, these methods are referred to as Strain Imaging (O'Donnell et al., 1994; Konofagou and Ophir, 1998; Lopata et al., 2011). In what follows we will use the term ST in a global sense to denote all those methods that estimate the motion by tracking the speckle pattern in B-mode US acquisitions.

The phenomenon of the speckle can be explained by the physics of the problem of wave transmission in a tissue in US: it is produced by the reflection of the transmitted coherent ultrasound waves at fixed frequencies for different tissues. Since this pattern remains stable under the same acquisition conditions and exhibits an inherent relationship with the tissue structure, it can be tracked to estimate the motion of the tissue (Trahey et al., 1986; Burckhardt, 1978).

Many different approaches to ST can be found in the literature, where they have extensively proved to be powerful tools in order to obtain quantitative and qualitative information regarding myocardial deformation, motion and function assessment (Notomi et al., 2005; Crosby et al., 2005; Suffoletto et al., 2006). The clinical relevance of the motion estimation in US B-mode images has motivated the community to improve the original ST technique proposed in Robinson et al. (1982) and Trahey et al. (1988) by more complex approaches. New contributions modify the original techniques in different ways, such as the statistical modeling of speckle, using more complex registration algorithms or applying different optimization algorithms for the ST estimation. It seems clear that modifications in different steps of the process will have a different impact over the results. In addition, the influence of certain methods may obliterate the complexity of previous steps. For instance, the use of certain registration algorithms could make the process highly invariant to the similarity measure used. Thus, it becomes necessary to identify the relevant relations between the different components involved in a ST method in order to clarify which component really improves the accuracy of the estimation of motion and strain, and which ones become redundant. That is, precisely, the aim and motivation behind this work.

Some previous studies about the performance of ST methods can be found in the literature. One of the first ones was carried out by Bohs and Trahey (1991), where authors showed that a classical block matching registration together with the sum of the absolute differences -a quite simple similarity measure- could be used instead of the correlation, originally proposed by Robinson et al. (1982), achieving similar tracking results and better performance. Friemel et al. (1995) extended this study to include the non-normalized cross-correlation. Moreover, they showed that there was no statistically significant difference between the normalized cross-correlation and the sum of absolute differences at different signal to noise ratios.

In contrast to these early performance studies, in this work we propose a more global and complete approach. Instead of studying the influence of a particular improvement, we will analyze the system as a whole, similarly to what was done in De Craene et al. (2013) and Curiale et al. (2015) for different ST methods. However, in the proposed study, we are taking into account the relation between the steps and different choices taken to implement a ST method. The starting point is a prior identification of the basic parts of a ST method. The influence of the different techniques that can be used in each of these parts will be quantitatively analyzed. The techniques considered for the study are the following:

1. Different models for US data representation, some of them assuming an underlying statistical model for the speckle.
2. Different registration philosophies, including the classic block matching and a demons approach (Thirion, 1998).

3. Different interpolation schemes such as nearest neighbor, linear and cubic.

4. The use of structural information into the deformation model by using the normalized convolution (Knutsson and Westin, 1993) and a maximum likelihood approach such as the one proposed in Curiale et al. (2015).

5. The use of different optimization techniques, such as coarseto-fine refinement or an efficient second-order minimization (ESM).

These techniques will not be independently analyzed since the influence of one over the other must also be taken into account and may provide useful insights for the development of novel ST methods.

This work presents two main contributions to the ST field. First, it provides a complete study to identify which are the components of a ST method with greater influence and impact over the motion and strain estimation accuracy. The second contribution is the methodology for the analysis of a ST system designed to provide an easy and systematic way to include other ST philosophies. The conclusions and recommendations obtained in this work are intended to serve as a reference about what can be expected when improving or introducing a new strategy into a ST system. We believe that new methodologies proposed for ST should not be analyzed isolatedly anymore, but in a holistic way considering the complete pipeline. Great improvements in certain steps of a ST method can be overpassed or undermined by the choices taken in further steps as we will show in the results of this work.

\section{Background}

Tracking the speckle patterns in US B-mode images was first reported by Robinson et al. (1982), where the authors introduced a method for determining the velocity of propagation of ultrasound in tissue by comparing individuals sectors from different transducer positions using the normalized cross-correlation. Inspired by this work, Trahey et al. (1988); 1987) proposed a novel technique of velocity imaging based upon measuring the direction and magnitude of local blood speckle pattern displacement in consecutive 2D B-mode images for blood flow detection. These displacements were estimated by using the most conceptually straightforward method, a block matching algorithm using the normalized crosscorrelation as the similarity measure.

Some other metrics were proposed following the same blockmatching methodology. Such is the case of Strintzis and Kokkinidis (1997), where a maximum likelihood (ML) methodology was used to provide a suitable metric for US images based on a multiplicative Rayleigh characterization. Strintzis and Kokkinidis (1997) showed that the classical ST method was improved when considering more adequate metrics. Likewise, Cohen and Dinstein (2002) extended the metric by including the relation between the multiplicative Rayleigh characterizations of consecutive frames, which resulted in a more accurate ST method. Those incremental improvements evidenced that a more detailed description of the speckle statistics provides more accurate ST methods when the block-matching methodology is adopted.

More elaborated ST approaches have been proposed to improve the ST focusing on the registration technique rather than the metric. However, it is important to remark that, though some registration or tracking techniques are used as a necessary step for ST, the speckle information present in US images should be coded into the similarity measure used for tracking. For instance, instead of the straightforward block matching, some authors proposed to introduce the speckle model into a Free-Form 


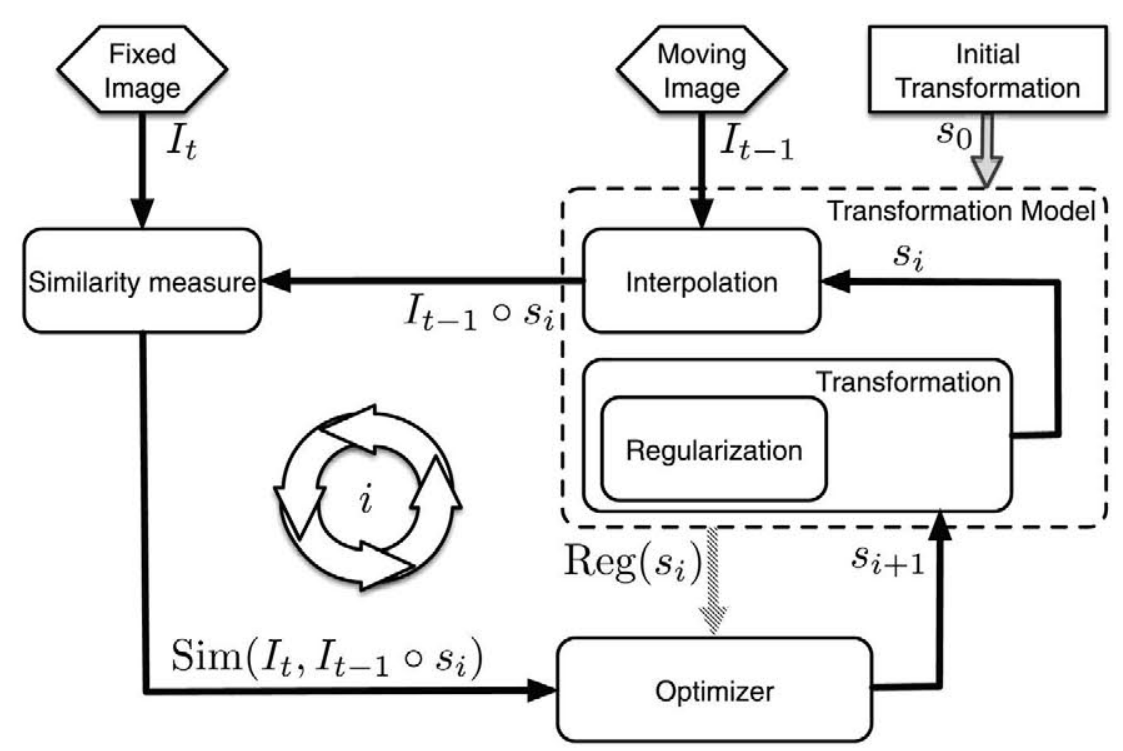

Fig. 1. Diagram of typical algorithms used in the intensity based registration methodology.

Deformation (FFD) method (Myronenko et al., 2009; Yue et al., 2009; De Craene et al., 2012; Curiale et al., 2013a), a diffeomorphic demons scheme (Curiale et al., 2015), or to introduce the monogenic phase of the B-mode image into an optical flow approach (Knutsson et al., 2005; Alessandrini et al., 2013; Tautz et al., 2013).

So, according to the proposals of the state of the art for ST, one can easily distinguish three necessary and interrelated parts within a generic ST method

1. Similarity measure: Necessary to establish the correspondence between images along the time sequence and may take advantage of speckle characterization in US images.

2. Transformation model: It specifies the type of motion considered between images and the way the similarity measure is introduced into the motion estimation.

3. Optimization strategy: It defines a strategy for optimizing the transformation model according to the similarity model.

These parts can be graphically depicted showing the generic flowchart of a ST method (Fig. 1). The diagram shows the three interrelated parts of an ST method as blocks whose inputs are the fixed image $I_{t}$, the moving image $I_{t-1}$, and an initial transformation $s_{0}$ that maps them. The initial transformation would act over the moving image by considering interpolation and regularization in order to ensure that every location in the moving image corresponds to a certain location in the fixed image. The performance of the resulting transformation is then measured by a similarity measure that can make use of the statistical characterization of speckle in US images. Finally, the optimizer tries to provide the best transformation according to the similarity measure. This process is commonly done in an iterative way until some stop rule is reached, though some approaches have just one iteration.

In what follows, the state of the art methods will be analyzed according to these parts with a special emphasis on the hypotheses assumed in their definition. A summary of the methods considered in this study and the techniques applied in each part of the ST methodology are shown in Table 1.

\subsection{Similarity measure}

The similarity measure is responsible for establishing a correspondence of similitude between two or more images; in the case of ST, images from a temporal sequence of an US acquisition. These similarity measures make use of the intensity or the speckle pattern, and some usually take advantage of the statistical description of speckle in US images. ST methods assume that the highest similarity is given when the optimal transformation is found.

During the past decades, different similarity measures have been proposed for ST, among them, the more extended are: the normalized cross-correlation (Robinson et al., 1982; Trahey et al., 1988; 1987); the sum of absolute differences (Bohs and Trahey, 1991; Bohs et al., 2000); the non-normalized crosscorrelation (Friemel et al., 1995); the sum of squared differences (Yeung et al., 1998a; 1998b; De Craene et al., 2012; Curiale et al., 2013b; Heyde et al., 2013a; 2013b; Piella et al., 2013; Somphone et al., 2013); the mutual information (Elen et al., 2008) and the monogenic phase (Knutsson et al., 2005; Alessandrini et al., 2013; Tautz et al., 2013).

On the other hand, some authors proposed the use of statistical models to provide a better characterization of speckle, such as Gaussian (Cohen and Dinstein, 2002), Rayleigh (Strintzis and Kokkinidis, 1997; Cohen and Dinstein, 2002; Yue et al., 2009), Gamma (Curiale et al., 2013a), bivariate Nakagami (Myronenko et al., 2009) and bivariate Generalized Gamma (Curiale et al., 2015).

Note that those similarity measures based on a statistical characterization of speckle are assuming that the deformation through time does not change the statistics of speckle, though the intensity values of the image may vary. Thus, the measures are intended to provide the most likely transformations according to the statistical characterization of speckle. So, the underlying hypothesis in the definition of these metrics is "the statistical characterization provides a better description of speckle that improves ST".

Besides, the metrics proposed by Myronenko et al. (2009) and Curiale et al. (2015) also considered a temporal correlation within the statistical model that accounts for correlation between speckle patterns throughout the deformation. These works assume that "temporal correlation leads to a better temporal tracking when statistical models of speckle are applied".

Others hybrid similarity measures such as the sum of squared differences combined with structural information (Myronenko et al., 2007) or the sum of squared differences combined with Rayleigh model (Piella et al., 2013) were proposed in order to jointly consider the statistical characterization of speckle and some local structural information assuming that "structural information 
Table 1

view of the most representative speckle tracking techniques and a few recent approaches. CC: Cross-Correlation, NCC: Non-normalized Cross-Correlation; SAD: Sum of absolute differences, SSD: sum of Square differences, SI: Structural Information, MP: Monogenic Phase, GG: Generalized Gamma, FFD: Free Form Deformation with B-Splines, BS: B-Splines, OF: Optical Flow, BM: Block Matching, NC: Normalized convolution. Not specified.

\begin{tabular}{|c|c|c|c|c|c|c|c|c|c|}
\hline \multirow[b]{2}{*}{ Author } & \multicolumn{2}{|l|}{ Similarity model } & \multicolumn{4}{|c|}{ Transformation model } & \multicolumn{3}{|c|}{ Optimization model } \\
\hline & Non-speckle model & Speckle model & Parametric & Non-parametric & $\mathrm{NC}$ & Temporal continuity & Non-iterative & Iterative & Multi-resolution \\
\hline Robinson et al. (1982) & $\mathrm{CC}$ & $x$ & $x$ & OF BM & $x$ & $x$ & $\sqrt{ }$ & $x$ & $x$ \\
\hline Trahey et al. (1988); 1987) & $\mathrm{CC}$ & $x$ & $x$ & OF BM & $x$ & $x$ & $\sqrt{ }$ & $x$ & $x$ \\
\hline Bohs and Trahey (1991) & SAD & $x$ & $x$ & OF BM & $x$ & $x$ & $\sqrt{ }$ & $x$ & $x$ \\
\hline Friemel et al. (1995) & $\mathrm{NCC} / \mathrm{CC} / \mathrm{SAD}$ & $x$ & $x$ & OF BM & $x$ & $x$ & $\sqrt{ }$ & $x$ & $x$ \\
\hline Strintzis and Kokkinidis (1997) & $x$ & Rayleigh & $x$ & OF BM & $x$ & $x$ & $\sqrt{ }$ & $x$ & $x$ \\
\hline Yeung et al. (1998a) & SSD & $x$ & $x$ & OF BM & $x$ & $x$ & $\sqrt{ }$ & $x$ & $\sqrt{ }$ \\
\hline Yeung et al. (1998b) & SSD & $x$ & $x$ & OF BM & $x$ & $x$ & $\sqrt{ }$ & $x$ & $\sqrt{ }$ \\
\hline Bohs et al. (2000) & SAD & $x$ & $x$ & OF BM & $x$ & $x$ & $\sqrt{ }$ & $x$ & $x$ \\
\hline Cohen and Dinstein (2002) & $x$ & Rayleigh & $x$ & OF BM & $x$ & $x$ & $\sqrt{ }$ & $x$ & $x$ \\
\hline Knutsson et al. (2005) & MP & $x$ & $x$ & OF & $x$ & $x$ & $x$ & $\mathrm{NC}$ & $\sqrt{ }$ \\
\hline Myronenko et al. (2007) & $\mathrm{SSD}+\mathrm{SI}$ & $x$ & FFD & $x$ & $x$ & $x$ & $x$ & Steepest descent & - \\
\hline Yue et al. (2009) & $x$ & Rayleigh & FFD & $x$ & $x$ & $x$ & $x$ & L-BFGS-B & $\sqrt{ }$ \\
\hline Myronenko et al. (2009) & $x$ & Bivariate Nakagami & FFD & $x$ & $x$ & $x$ & $x$ & Steepest descent & $\sqrt{ }$ \\
\hline De Craene et al. (2012) & SSD & $x$ & FFD & $x$ & $x$ & $\sqrt{ }$ & $x$ & L-BFGS-B & $\sqrt{ }$ \\
\hline Curiale et al. (2013a) & $x$ & Gamma & FFD & $x$ & $x$ & $x$ & $x$ & Conjugate gradient & $\sqrt{ }$ \\
\hline Curiale et al. (2013b) & SSD & $x$ & $x$ & Diffusion & $\sqrt{ }$ & $x$ & $x$ & Variational & $\sqrt{ }$ \\
\hline Heyde et al. (2013b) & SSD & $x$ & FFD & $x$ & $x$ & $x$ & $x$ & L-BFGS-B & $\sqrt{ }$ \\
\hline Tautz et al. (2013) & MP & $x$ & $x$ & OF & $x$ & $x$ & $x$ & $\mathrm{NC}$ & $\sqrt{ }$ \\
\hline Heyde et al. (2013a) & SSD & $x$ & FFD & $x$ & $x$ & $x$ & $x$ & L-BFGS-B & $\sqrt{ }$ \\
\hline Somphone et al. (2013) & SSD & $x$ & $x$ & Diffusion & $\sqrt{ }$ & $x$ & $x$ & Steepest descent & $\sqrt{ }$ \\
\hline Alessandrini et al. (2013) & MP & $x$ & $x$ & OF with BS & $x$ & $x$ & $x$ & Variational & $\sqrt{ }$ \\
\hline Piella et al. (2013) & SSD & Rayleigh & FFD & $x$ & $x$ & $x$ & $x$ & L-BFGS-B & $\sqrt{ }$ \\
\hline Curiale et al. (2015) & $x$ & Bivariate GG & $x$ & Diffusion & $\sqrt{ }$ & $x$ & $x$ & Variational & $\sqrt{ }$ \\
\hline
\end{tabular}


provides a better description of the deformation than just the statistical characterization".

\subsection{Transformation model}

The transformation model defines the sort of deformation allowed for the ST technique, and the way the similarity measure is evaluated. A well-known example is a rigid or affine transformation. However, this transformation is too restrictive to model complex motions such as heart motion, which are better described by using non-rigid transformations. ${ }^{1}$ The non-rigid transformations could be split in two main groups according to the way the deformation is described: function representations and physical based models.

Function representations make use of a small number of parameters and much of the mathematical framework arises from the theory of function interpolation and approximation theory. Thus, they are also known as parametric models. One good example is the Free-Form-Deformation (FFD) model, where motion is parametrized by smooth function such a B-splines. In this model, the control points and spline functions are used to establish locally the correspondence between images. The FFD is computationally efficient and has been widely used in ST methods (Myronenko et al., 2007; 2009; Yue et al., 2009; De Craene et al., 2012; Curiale et al., 2013a; Heyde et al., 2013a; 2013b; Piella et al., 2013) due to its computational efficiency.

Physical based models are dense and non-parametric transformations, where the displacement field is defined in each voxel (Holden, 2008) according to physical phenomena. Such is the case of elastic models (Bajcsy and Kovačič, 1989), fluid models (Beauchemin and Barron, 1995), optical flow and diffusion models (Thirion, 1998). All these transformation models assume that the intensity level of corresponding voxels remains the same when the images are deformed.

Elastic models treat the image as a linear elastic model and deform it using forces derived from an image similarity measure. In this context, the image is deformed until an equilibrium state is reached. Since the linear elasticity assumption is only valid for small deformations, it is hard to recover large image differences. To overcome this problem, the elastic model is replaced by a fluid model which allows large and highly localized deformation.

Optical flow registration is widely used in speckle tracking and it can be divided in two general methods: block matching methods (Robinson et al., 1982; Trahey et al., 1988; 1987; Bohs and Trahey, 1991; Friemel et al., 1995; Strintzis and Kokkinidis, 1997; Yeung et al., 1998a; 1998b; Bohs et al., 2000; Cohen and Dinstein, 2002; Leitman et al., 2004) and variational methods (Knutsson et al., 2005; Curiale et al., 2013b; 2015; Alessandrini et al., 2013; Somphone et al., 2013; Tautz et al., 2013).

The diffusion or demons model introduced by Thirion (1998) can be thought as an approximation to fluid registration. Vercauteren et al. (2009) showed that the demons algorithms could be seen as an optimization procedure for a global energy on the entire space of displacement fields. Also, they showed that demons algorithm could be adapted to provide a non-parametric diffeomorphic transformation with a second-order minimization. Recently, Curiale et al. (2015) adapted the diffeomorphic demons method for strain estimation in 3D echocardiography by introducing a Generalized Gamma speckle model into the global energy to be minimized.

The transformation model also comprises the interpolation used when a point is mapped from one space into another by certain

\footnotetext{
${ }^{1}$ An interesting review of geometric transformations for non-rigid body can be found in Holden (2008).
}

transformation, i.e. image values in non-integer coordinates are computed by the appropriate interpolation technique. The interpolation used can affect the accuracy and convergence of ST. To decrease the computational time, a simple scheme such as nearest neighbor or linear interpolation is usually applied in the optimization. More complex interpolation schemes can be used for higher accuracy, among them, cubic, B-spline or sinc interpolation functions.

Obviously, any interpolation affects to any speckle model applied in combination to any of the non-rigid transformations. However, most of the methods applying speckle models assume that "interpolation has a negligible effect in the ST accuracy".

Finally, there are different approaches to constrain the transformation model with the main goal of improving the ST accuracy. Some authors, for instance, introduce the temporal consistency into the transformation model (De Craene et al., 2012), while others make use of an extra regularization by using the normalized convolution proposed by Knutsson and Westin (1993) in order to constrain the transformation to relevant tissue (Curiale et al., 2013b; 2015; Somphone et al., 2013).

\subsection{Optimization strategy}

The motion field or tracking between two or more objects inside the image is found by optimizing the transformation model according to the similarity measure.

This optimization can be done by using many methods, such the classical gradient descent or the steepest descent optimization (Myronenko et al., 2007; 2009), the conjugate gradient (Curiale et al., 2013a), the Broyden Fletcher Goldfarb Shannon (L-BFGS-B) (Yue et al., 2009; De Craene et al., 2012; Heyde et al,, 2013a; 2013b; Piella et al., 2013) or the normalized convolution (Knutsson et al., 2005; Tautz et al., 2013) among others. Fluid, elastic and diffusion models that can be described in terms of partial differential equations are commonly optimized by using variational approaches (Alessandrini et al., 2013; Somphone et al., 2013; Curiale et al., 2013b; 2015). All these methods are well documented in Press et al. (2007).

Furthermore, a widely used strategy for improving the optimization, especially for large deformations, is to use a coarse-tofine refinement (Yeung et al., 1998a; 1998b; Knutsson et al., 2005; Yue et al., 2009; Myronenko et al., 2009; De Craene et al., 2012; Curiale et al., 2013a; 2013b; Heyde et al., 2013b; 2013a; Somphone et al., 2013; Alessandrini et al., 2013; Piella et al., 2013; Tautz et al., 2013). This approach is highly recommended for the complex deformations such is the case of the heart motion. Thus, it is expected that "a coarse-to-fine refinement improves the ST accuracy".

\section{Materials and methods}

As stated in the previous section, a complete ST procedure is conformed by three different parts that can be implemented using many different methods. Alongside, for each method, different design options may be considered. Therefore, a practical implementation should involve many different choices that can affect the accuracy of the tracking. The purpose of this work is precisely to analyze the influence of the different steps and design options over the global performance of the ST. Since it would be unpractical to consider all possible combination of parameters, we will take as starting point the hypotheses derived from the main results reported in the literature, some of them already introduced in Section 2. A set of experiments is defined to properly test the validity and extension of the initial hypotheses following the methodology here described. 


\subsection{Methodology}

The analysis of the influence of the multiple techniques used in ST methods is done according the to the different possible choices described in Section 2 within the three components of a generic ST method: the similarity measure, the transformation model and the optimization strategy (depicted in Fig. 1). The proposed analysis will test the underlying hypotheses that have been identified from the methods of the state of the art:

Hypothesis 1. Similarity measures based on a speckle model characterization are more reliable to real US data and improve the ST accuracy.

Hypothesis 2. Speckle models that take into account temporal correlation improve ST accuracy.

Hypothesis 3. Although interpolation modifies the statistical model assumed for characterizing the speckle pattern, it has a negligible effect on the ST accuracy.

Hypothesis 4. The transformation model has greater influence on the ST accuracy than the similarity measure.

Hypothesis 5. Structural information increases the ST accuracy more than just the speckle model.

Hypothesis 6. A coarse to fine refinement approach improves significantly the ST accuracy for complex motions.

Hypothesis 7. A complex interpolation scheme improves the ST accuracy, regardless of the similarity measure selected.

Hypothesis 8. The optimization strategy has more influence in the ST accuracy than the similarity measure.

In addition, note that there are not initial assumptions about the cross performance of improvements in different steps of the procedure, such as a better transformation model with structural information and a coarse to fine refinement. This interrelations are the key of the buildup of an efficient and accurate ST pipeline, and therefore they must also be analyzed.

In order to validate these hypotheses, a series of experiments will be conducted. Due to the impracticability of considering all the possible combinations of methods and parameters reported in literature, we will confine ourselves only to those relevant and representative for each of the three steps defined for ST. The analysis of the similarity measure will consider the sum of squared differences (SSD) and other metrics based on speckle characterization (summarized in Table 1) in order to analyze the influence of the speckle statistical models on the ST performance.

On the other hand, two nonparametric transformation models will be considered in order to minimize the influence of the optimization strategy into the final estimation. We decided to study the classic block matching and the diffusion approaches since the number of strategies used to optimize the transformation model and the number of free parameters to fix are reduced.

The quantitative evaluation will be performed by considering a realistic synthetic data as a Gold standard that will be described in Section 3.2. The accuracy of the ST methods will be evaluated by the measuring the error (Euclidean distance) for motion and strain estimation with the ground truth.

In all the cases, the procedure to evaluate one particular hypothesis will be similar: a simple ST pipeline will be considered. Different configurations are used only for the method or strategy under study, i.e. that one implied by the current hypothesis. The procedures for the other methods are fixed in a simple configuration, so that it allows identifying the actual influence of the parameter under study over the global performance. This methodology will be adjusted to the particularity of each of the analyzed choices, and it will be described in detail for each experiment in Section 4.

First, according to hypotheses 1 and 2, the influence of the similarity measure is analyzed. In particular, the analysis of the influence of the similarity measure takes into account the measures described in Table 2.

Second, following hypotheses $[3,4,5]$ and 7 , the influence of the transformation model is studied. We will consider the following strategies:

- Transformation: Two different nonparametric transformations are taken into account: The classical Block Matching and a diffeomorphic diffusion or Demons approach (Vercauteren et al., 2009). The reader can refer to Curiale et al. (2015) for more details about the demons implementation.

- Interpolation: Three different interpolation types are considered: nearest neighborhood, linear and cubic interpolation.

- Regularization: The influence of myocardial structural information is analyzed by using the normalized convolution to reduce the number of possible transformations.

Finally, consistent with hypotheses 6 and 8, we evaluate the influence of the optimization strategy, specifically taking into account an iterative and non-iterative approach. The optimization strategy used for the block matching algorithm is based on finding the best correspondence within a set of blocks defined by the search radius, so it is a non-iterative approach and does not require extra interpolations. In contrast, the optimization strategy used for the diffusion-based approach makes use of a variational formulation which is iteratively refined and requires several interpolations. Thus, we expect to observe different behaviors between the block matching and the demons approach for the similarity models. Furthermore, we study the use of an efficient second-order minimization for the demons approach and a coarse to fine refinement for both transformation models.

Along all the experiments, we decide to prioritize the temporal coherence along the cardiac phase instead of avoiding the frame to frame error accumulation. Therefore, the registration algorithms were applied frame by frame between two consecutive images in the cardiac phase. During the derivation of the speckle tracking methods the parameters for the Gamma, Nakagami and Generalized Gamma distributions were estimated. The Gamma and Generalized Gamma parameters are estimated by means of the Gamma and Generalized Gamma Mixture Model (Vegas-SanchezFerrero et al., 2012; 2014). On the other hand, the temporal correlation of the Nakagami and Generalized Gamma distribution is estimated as it was proposed by Curiale et al. (2015).

\subsection{Materials}

The quantitative evaluation of the different methods is carried out by using a synthetic cardiac US data set: the public benchmark named Straus (De Craene et al., 2013). The use of synthetic data is motivated by the need of a Gold Standard that allows a numerical comparison of the actual values of strain and motion. This way, we can quantify the degree of influence of each of the studied methods over the final estimation, a task that could not be done by using real data sets.

The Straus dataset provides a realistic cardiac 3D geometry of $297 \times 297 \times 297$ pixels with an isotropic resolution of 0.3367 $\mathrm{mm}$ (Fig. 2). The ultrasound images were obtained from the segmentation of cine magnetic resonance images, which were used to simulate conventional US images. This dataset provides a realistic cardiac geometry with three different complex motions: normal (no lesion), acute ischemia and acute dyssynchrony. The ultrasound speckle structure was synthetically generated by using a sampling at $50 \mathrm{MHz}$ with a phased array transducer centered at $3.3 \mathrm{MHz}$ 
Table 2

Summary of the similarity measures studied. Constants $m$ and $\beta$ are shape parameters of the Generalized Gamma distribution; $\alpha$ is the shape parameter of the Gamma distribution and $\rho$ is the correlation between different time frames $I_{t-1}$ and $I_{t}$ (details on these similarity measures can be found in (Curiale et al., 2015)).

\begin{tabular}{|c|c|c|}
\hline Relationship & Similarity measure & References \\
\hline \multirow[t]{9}{*}{$z=I_{t-1}-I_{t}$} & $\mathrm{SSD}=\|z\|^{2}$ & Yeung et al. (1998a); 1998b); Myronenko et al. (2007) \\
\hline & & Curiale et al. (2013b); Heyde et al. (2013a); 2013b) \\
\hline & & Piella et al. (2013); Somphone et al. (2013) \\
\hline & $\mathrm{CD} 2=\log (\exp (2 z+1))-z$ & Strintzis and Kokkinidis (1997); Cohen and Dinstein (2002) \\
\hline & & Yue et al. (2009); Piella et al. (2013) \\
\hline & $\mathrm{MS} 2=(m+0.5) \log \left(\cosh ^{2}(z)-\rho\right)$ & Myronenko et al. (2009) \\
\hline & $-0.5 \log \left(\cosh ^{2}(z)\right)$ & \\
\hline & $\mathrm{GGCS}=(m+0.5) \log \left(\cosh ^{2}(z \beta)-\rho\right)$ & Curiale et al. (2015) \\
\hline & $-0.5 \log \left(\cosh ^{2}(z \beta)\right)$ & \\
\hline \multirow[t]{3}{*}{$z=I_{t-1} / I_{t}$} & $\mathrm{GS}=\alpha \log \left(z+z^{-1}+2\right)$ & Curiale et al. (2013a) \\
\hline & $G G S=(m+0.5) \log \left(\left(z^{2 \beta}+1\right)^{2}-4 \rho z^{2 \beta}\right)$ & \\
\hline & $-2 \beta m \log (z)-\log \left(z^{2 \beta}+1\right)$ & \\
\hline
\end{tabular}
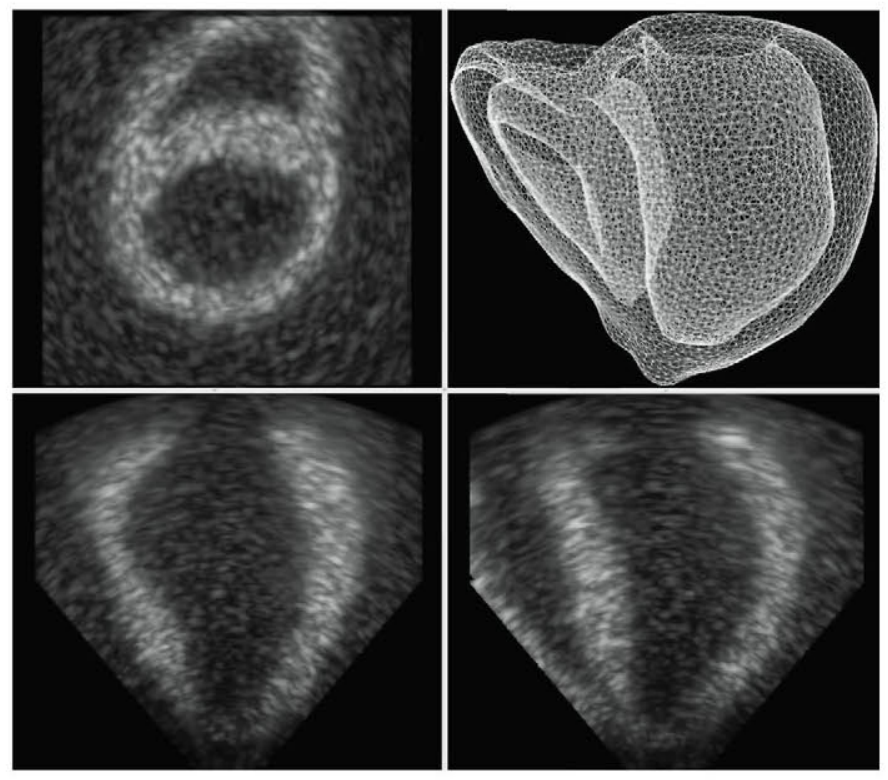

Fig. 2. Synthetic ultrasound images generated by Straus. The 3D synthetic US images are presented in three orthogonal views. The shape model is depicted in the top-right corner.

and by transmitting a Gaussian pulse with a $-6 \mathrm{~dB}$ relative bandwidth of $65 \%$. A symmetric transverse two-way beam profile was assumed, focusing at $80 \mathrm{~mm}$. The simulated images consisted of $107 \times 80$ lines in azimuth and elevation direction over an angle of $80 \times 80$ degrees, resulting in a frame rate of $30 \mathrm{~Hz}$ due to the use of parallel beam forming. In this way, the cardiac phase for the normal case comprises 26 images. A detailed description of the data setup can be found in De Craene et al. (2013).

The Straus dataset also provides several image sequences of the normal case with different signal to noise (SNR) levels. These cases were generated to simulate the SNR levels by modifying the relative amplitude of tissue with respect to blood pool scatters. This change means an increasing noise power and it is directly related with a contrast value (CNR) according to the equation (Wijk and Thijssen, 2002):

$\mathrm{CNR}=\frac{\mu_{T}-\mu_{B}}{\sqrt{\left(\sigma_{B}^{2}+\sigma_{T}^{2}\right) / 2}}$,

where $\mu_{T}$ and $\mu_{B}$ are the average intensities value of the tissue and blood pool, and $\sigma_{T}^{2}$ and $\sigma_{B}^{2}$ are their variances respectively. The quantitative evaluation for the proposed methodology was carried out only using the normal case without pericardium to avoid the influence of pathological cases and the pericardium in the ST technique. For robustness assessment of the ST techniques at different SNR levels, three different contrast to noise ratio values were used, $\mathrm{CNR}=\{1.2,1.8,2.5\}$ (Fig. 3 ).

The data set provides changes in the ultrasound image intensity throughout the cardiac cycle since it makes use of a new speckle pattern realization for each 3D ultrasound b-mode image. This way, they show a more realistic behavior than considering transformations over the same intensity levels across frames.

For the parameter settings of the speckle tracking methodologies, the diffeomorphic demons approach is applied to the synthetic dataset with the parameters setting proposed by Curiale et al. (2015), and the block matching approach makes use of a block size of $3 \times 3 \times 3$ pixels within a search region of $3 \times 3 \times 3$ pixels. The demons approach is applied by using nLevels $=3$ with a maximum of 30 iterations per level (multi-resolution strategy) or when the difference between deformations is below a tolerance $\mathrm{TOL}=10^{-7}$. The standard deviation values used in the regularization were determined using an independent linear search between $[1,8] \mathrm{mm}$ for the regularization and $[0.5,5.5] \mathrm{mm}$ for the maximum step. On the other hand, the kernel sizes were selected as a tradeoff between the motion and strain accuracy and the total runtime for the Generalized Gamma mixture model. The reader can refer to Curiale et al. (2015) for the optimized values used in the demons approach.

This configuration is maintained throughout the experiments in order to see the influence of the different choices of similarity measures, transformation models and optimization techniques without the influence of uncontrolled confounding factors.

\section{Experiments and results}

According to the methodology proposed, a set of experiments is defined to properly test the validity and extension of the different hypotheses defined in the previous section. In Table 3 we show a summary of the experiments and their relations with the hypothesis tested as follows: First, an analysis of the effect of the similarity measures and transformation models are done by studying their influence in the motion and strain accuracy. Then, the interpolation scheme, the myocardial structural information and the multi-resolution approach are analyzed. Finally, the performance of the complete ST pipeline is studied by evaluating the motion and strain accuracy. 

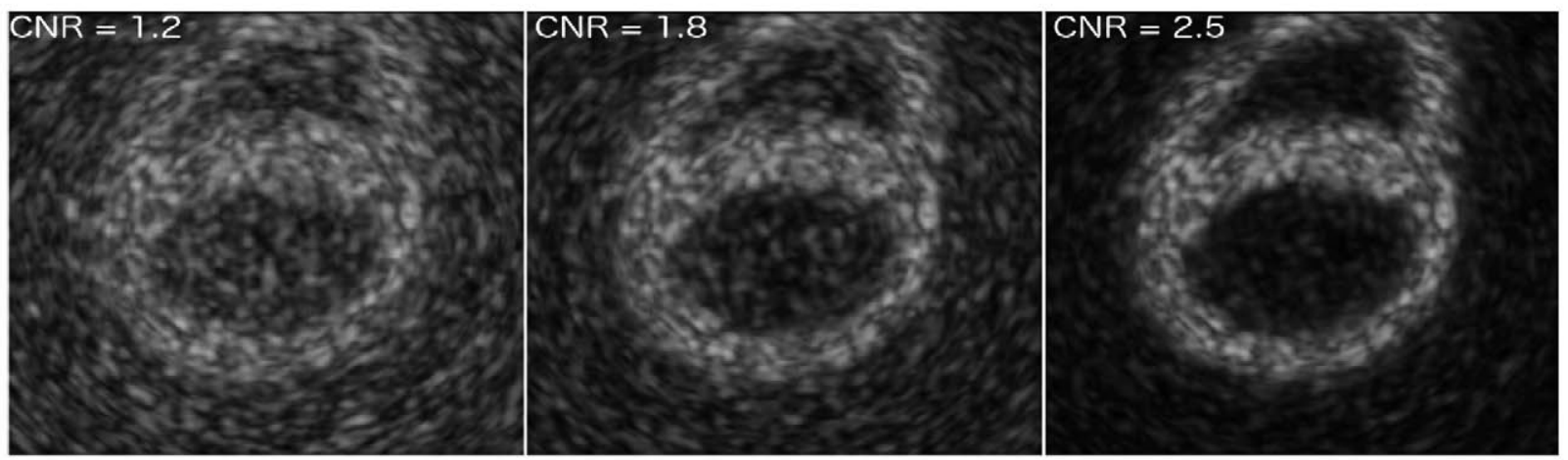

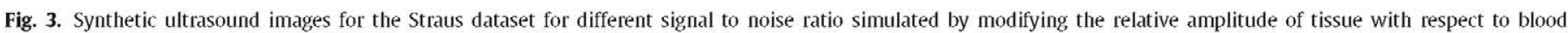
pool scatters (CNR). The 3D synthetic US images are presented in the short axis view.

Table 3

Summary of the experiments. BM: Block Matching. Int.: Interpolation type, NN: nearest neighborhood, L: linear, C: Cubic. Struct. Inf.: myocardial structural information. MR: multiresolution, ESM: efficient second order minimization. -: Whole pipeline experiment.

\begin{tabular}{|c|c|c|c|c|c|c|c|c|}
\hline & \multirow{2}{*}{$\begin{array}{l}\text { Similarity } \\
\text { Measures }\end{array}$} & \multicolumn{4}{|c|}{ Transformation } & \multicolumn{2}{|c|}{ Optimization } & \multirow[t]{2}{*}{ Hyp. } \\
\hline & & $\mathrm{BM}$ & Demons & Int. & Struct. Inf. & MR & ESM & \\
\hline Exp. 1 & All & $\sqrt{ }$ & $\sqrt{ }$ & $\mathrm{L}$ & $x$ & $x$ & $x$ & $1,2,4$ \\
\hline Exp. 2 & All & $\sqrt{ }$ & $\sqrt{ }$ & $\mathrm{NN} / \mathrm{L} / \mathrm{C}$ & $x$ & $\sqrt{ }$ & $x$ & 3,7 \\
\hline Exp. 3 & All & $\sqrt{ }$ & $\sqrt{ }$ & $\mathrm{L}$ & $\sqrt{ }$ & $x$ & $x$ & 5 \\
\hline Exp. 4 & All & $\sqrt{ }$ & $\sqrt{ }$ & $\mathrm{L}$ & $x$ & $\sqrt{ }$ & $\sqrt{ }$ & 6,8 \\
\hline Exp. 5 & All & $\sqrt{ }$ & $\sqrt{ }$ & $\mathrm{L}$ & $\sqrt{ }$ & $\sqrt{ }$ & $\sqrt{ }$ & - \\
\hline
\end{tabular}

\subsection{Effect of similarity measures and transformation models}

In the following experiment, we evaluate Hypotheses 1 and 2 by studying the influence of the similarity measures and speckle models (gathered in Table 2) by testing the performance of the different similarity measures for motion and strain estimation. Also, this experiment evaluates the Hypothesis 4 to study the influence of the transformation model over the ST accuracy.

Experiment 1. The errors in motion and strain are analyzed for the Strauss data set for all the proposed similarity measures. A classic block matching algorithm and a simple diffeomorphic demons approach are used, (i.e. without myocardial structural information or multi-resolution strategy). The results were statistically analyzed by a sign test and a bootstrapping strategy with two hundred repetitions applied to a set of 100 error samples for each frame in the cardiac phase. This statistical test was chosen due to the lack of symmetry on the error difference between similarity measures for motion and strain.

Results of the experiments are gathered in Table 4 (average error along the cardiac phase). The results for the block matching approach show a subtle but still statistically significant difference between metrics based on intensity ratios (GS and GGS around $3.08 \mathrm{~mm}$ ) and metrics based on intensity differences (CD2, MS2, GGCS2, around $3.15 \mathrm{~mm}$ ) due to the inclusion of log-compression into the metric derivation ( $p$-value $<10^{-4}$ in all cases). This differences were expected since the similarity measures GS and GGS consider spatial and temporal correlation between images, however the lower performance of GGCS compared to the GGS is due to the assumption of log-compression in the metric. Note that the inclusion of log-compression in this metrics implicitly assumes that intensities are actually log-compressed intensities, and the parameters of the characterization must be estimated considering an idealistic logarithmic compression, whose parameters are also unknown. This fact introduces an additional confounding factor that affects negatively to the motion estimation. Additionally, the SSD metric -which does not assume any statistical model- performs as good as the best statistical methods $(3.08 \mathrm{~mm})$, though it does not perform as good with the circumferential strain (13.39\% for SSD vs. $19.06 \%$ for GS with $p$-value $<10^{-2} ; 18.64 \%$ for GS with a $p$-value $\left.<10^{-4}\right)$. This result means that the coarse deformation can be perfectly estimated with SSD. However, a more detailed metric that accounts for spatial and temporal correlation is needed to describe circumferential deformations.

As a conclusion, we can state that both Hypotheses 1 and 2 are confirmed for the block matching approach since spatial and temporal correlations increase the accuracy, but the log-compression assumption on the intensity labels introduce some additional uncertainty in the estimation which penalizes the estimation.

Interestingly, there is an apparent decrease of the performance of metrics GS and GGS for the radial strain error (over $24 \%$ ). This is due to the effect that most of the deformation is performed in the radial direction, which makes the radial estimation more prone to a higher number of candidates in the search window. The ones selected by these metrics are those providing a lower ratio of intensities, which is more sensitive to variations than the simple difference of intensities. As we will see in further experiments, the optimization techniques will be of great importance when these similarity measures are used and this undesired effect can be effectively avoided by using a more suitable transformation model like demons. The results obtained for demons show a great performance compared to the block matching method as expected. In this case, the measures that perform the best are those that consider the logarithmic compression into the model (CD2, MS2 and GGCS) with motion error around $0.78 \mathrm{~mm}$, however those not assuming compression (GS and GGS) obtain the most accurate strain estimate and both groups are statistically different ( $p$-value $<10^{-3}$ in all the comparisons between metrics from different groups).

This apparent contradiction can be explained from the analysis of both transformation techniques in combination with the different metrics. Note that the more sophisticated statistical models which account for spatial and temporal correlations in the speckle model (GS, GGS) provide a better estimate of the deformation when isolated blocks are considered. This is confirmed by 
Table 4

Quantitative results of Experiment 1 for average motion and strain accuracy using block matching (BM) and the simple demons approach, i.e. without a coarse-to-fine refinement and without the normalized convolution.

\begin{tabular}{|c|c|c|c|c|c|c|c|c|c|}
\hline \multirow[b]{2}{*}{ CNR } & \multirow[b]{2}{*}{ Similarity } & \multicolumn{2}{|c|}{ Motion Error $(\mu)[\mathrm{mm}]$} & \multicolumn{2}{|c|}{ Circ. strain error $(\mu)[\%]$} & \multicolumn{2}{|c|}{ Long. strain error $(\mu)[\%]$} & \multicolumn{2}{|c|}{ Rad. strain error $(\mu)[\%]$} \\
\hline & & BM & Demons & $\mathrm{BM}$ & Demons & BM & Demons & BM & Demons \\
\hline \multirow[t]{6}{*}{2.5} & SSD & 3.08 & 0.78 & 19.35 & 2.29 & 13.66 & 2.76 & 23.42 & 20.64 \\
\hline & $\mathrm{CD} 2$ & 3.14 & 0.79 & 19.32 & 2.29 & 13.95 & 2.76 & 23.31 & 20.86 \\
\hline & MS2 & 3.15 & 0.78 & 19.38 & 2.28 & 13.96 & 2.76 & 23.27 & 20.25 \\
\hline & GGCS & 3.15 & 0.79 & 19.40 & 2.28 & 13.96 & 2.76 & 23.27 & 20.43 \\
\hline & GS & 3.08 & 0.93 & 19.06 & 2.15 & 13.52 & 2.45 & 24.56 & 13.83 \\
\hline & GGS & 3.07 & 0.93 & 18.64 & 2.15 & 13.34 & 2.45 & 24.09 & 13.64 \\
\hline \multirow[t]{6}{*}{1.8} & SSD & 3.14 & 1.02 & 19.04 & 2.64 & 14.22 & 3.13 & 23.88 & 26.50 \\
\hline & $\mathrm{CD} 2$ & 3.20 & 1.03 & 18.97 & 2.64 & 14.45 & 3.13 & 23.58 & 26.59 \\
\hline & MS2 & 3.21 & 1.02 & 19.06 & 2.64 & 14.46 & 3.13 & 23.53 & 26.26 \\
\hline & GGCS & 3.21 & 1.03 & 18.91 & 2.63 & 14.48 & 3.12 & 23.52 & 26.31 \\
\hline & GS & 3.14 & 1.23 & 18.91 & 2.60 & 14.11 & 2.81 & 25.22 & 17.67 \\
\hline & GGS & 3.14 & 1.23 & 18.57 & 2.60 & 13.87 & 2.81 & 24.50 & 17.54 \\
\hline \multirow[t]{6}{*}{1.2} & SSD & 3.19 & 1.22 & 18.97 & 2.85 & 14.68 & 3.60 & 25.30 & 30.36 \\
\hline & $\mathrm{CD} 2$ & 3.26 & 1.24 & 19.03 & 2.85 & 14.94 & 3.60 & 24.85 & 30.38 \\
\hline & MS2 & 3.26 & 1.23 & 19.01 & 2.84 & 14.93 & 3.59 & 24.82 & 30.14 \\
\hline & GGCS & 3.26 & 1.24 & 19.02 & 2.84 & 14.92 & 3.59 & 24.83 & 30.11 \\
\hline & GS & 3.20 & 1.54 & 18.82 & 3.23 & 14.60 & 3.26 & 25.96 & 21.14 \\
\hline & GGS & 3.20 & 1.54 & 18.60 & 3.23 & 14.33 & 3.26 & 24.99 & 21.03 \\
\hline
\end{tabular}

the lower motion error in block matching and also by the lower circumferential and longitudinal strain error. However, the block matching method reduces its efficiency with large deformations, causing inaccurate estimation of radial strain. When a more suitable technique which considers diffeomorphic transformation and spatial regularization is used to provide a more accurate motion estimation, the influence of the metrics is reduced to increase the spatial continuity. This is the case of metrics whose intensities are related with a difference such as the case of sum of squared differences (SSD) due to the log-compression assumption in the statistical model (CD2, MS2, GGCS). However, if the statistical method does not consider the log-compression (GS and GGS) the motion estimation is reduced due to the bigger fluctuations caused by the ratio of intensities, though the strain is generally better calculated because the inner regularization scheme used in the demons method includes a more suitable speckle model, which prevents from an excess of regularization (i.e. the motion estimation is more noisy but less biased by other non-related nearby motions resulting in a better estimate of strain).

The analysis of different regularization schemes will be studied in the following experiments, where we will consider multiresolution refinements that will reduce the excess or regularization by analyzing the images at different scales. With the multiresolution schemes, the metrics GS and GGS should show a lower motion error due to the iterative refinements at different resolutions which will provide better motion estimates in the radial direction (the largest deformation) without causing an excess of regularization that may cause low strain estimation performance. It is also important to note that there is an interdependence between the influence of the similarity measure and the transformation/optimization model, which is a side effect of the operations involved in the metric and the relationship of the moving image and the fixed image. This effect will be further investigated in the section named "Discussion on the interdependences".

So far, we have shown that the similarity measures have certain influence in each transformation model separately. However, if we consider the deformation model itself for both approaches, it remains clear that a more accurate deformation model obtains better results for both motion and strain estimates. As an example, Fig. 5 shows the motion errors along the cardiac phase for both approaches, block matching and demons, using a simple similarity measure (SSD) for all the CNR considered. Likewise, Fig. 4 shows the spatial circumferential strain error for three speckle models

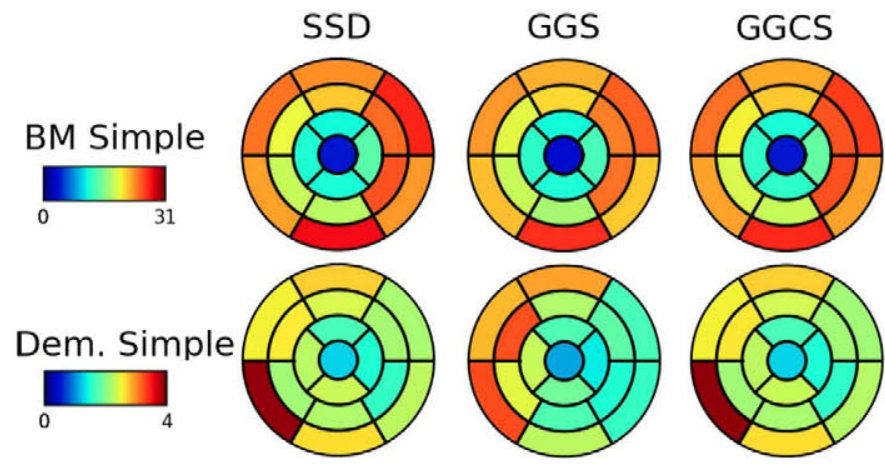

Fig. 4. Global circumferential strain accuracy for the squared sum of differences (SSD) and the Generalized Gamma similarity measures with and without compres sion (GGCS and GGS) for the Straus dataset within the 17 segment model at end systole and $\mathrm{CNR}=2.5$.

(SSD, GGS, GGCS) within the 17-segments model AHA. These results confirm the importance of the transformation model for motion and strain ST accuracy, which confirms the Hypothesis 4.

\subsection{Effect of interpolation and transformation models}

The deformation defined by the transformation model implies an interpolation of intensities in one of the images. Different interpolation strategies can be adopted which may show different results on the ST accuracy, as Hypothesis 3 states. To analyze this influence, the following experiment is done:

Experiment 2: The motion and strain accuracy are evaluated by using three different interpolation strategies: nearest neighborhood, linear and cubic. Both transformation methods are considered together with a multi-resolution optimization strategy.

The multi-resolution approach is applied in this experiment to increase the number of interpolation steps to amplify the influence of different interpolation schemes and to analyze the performance of similarity measures when the regularization schemes are more accurate. The differences between interpolation types were statistically analyzed, as in the previous experiment.

Table 5 shows the average motion and circumferential strain error at different noise levels (CNR $=\{1.2,2.5\}$ ). Longitudinal and radial strain errors showed similar behavior and were omitted for the sake brevity. Results do not show any significant 

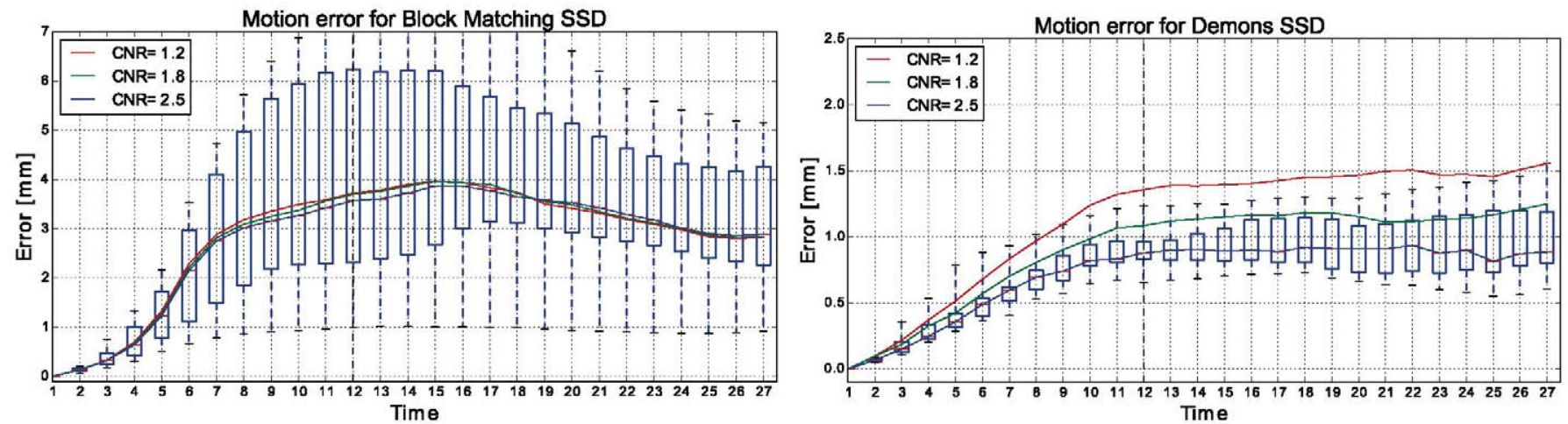

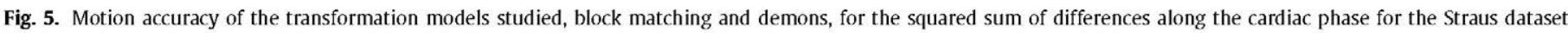
to different signal to noise ratio levels $C N R \in[1.2,1.8,2.5]$. The cardiac frame at end-systole is shown as a vertical dash line.

Table 5

Global average motion and strain error for the Straus dataset by interpolation types: nearest neighborhood, linear and cubic.

\begin{tabular}{|c|c|c|c|c|c|c|c|c|c|c|c|c|c|}
\hline \multirow[b]{3}{*}{ CNR } & \multirow[b]{3}{*}{ Similarity } & \multicolumn{6}{|c|}{ Average motion error, $\mu$ [mm] } & \multicolumn{6}{|c|}{ Average circumferential strain error, $\mu$ [\%] } \\
\hline & & \multicolumn{3}{|c|}{ Block matching } & \multicolumn{3}{|l|}{ Demons } & \multicolumn{3}{|c|}{ Block matching } & \multicolumn{3}{|l|}{ Demons } \\
\hline & & Nearest & Linear & Cubic & Nearest & Linear & Cubic & Nearest & Linear & Cubic & Nearest & Linear & Cubic \\
\hline \multirow[t]{6}{*}{2.5} & SSD & 0.98 & 0.96 & 0.96 & 0.83 & 0.74 & 0.76 & 18.91 & 18.95 & 18.80 & 2.65 & 2.29 & 2.31 \\
\hline & $\mathrm{CD} 2$ & 0.99 & 0.97 & 0.96 & 0.84 & 0.75 & 0.78 & 19.15 & 18.95 & 18.74 & 2.66 & 2.29 & 2.28 \\
\hline & MS2 & 0.99 & 0.97 & 0.96 & 0.83 & 0.74 & 0.77 & 19.07 & 18.99 & 18.69 & 2.67 & 2.27 & 2.26 \\
\hline & GGCS & 0.99 & 0.97 & 0.96 & 0.83 & 0.75 & 0.78 & 19.04 & 18.92 & 18.69 & 2.66 & 2.27 & 2.25 \\
\hline & GS & 1.01 & 0.98 & 0.98 & 0.79 & 0.70 & 0.73 & 18.54 & 18.43 & 18.09 & 2.26 & 1.92 & 1.92 \\
\hline & GGS & 1.01 & 0.99 & 0.99 & 0.79 & 0.70 & 0.73 & 17.71 & 17.72 & 17.45 & 2.28 & 1.92 & 1.92 \\
\hline \multirow[t]{6}{*}{1.2} & SSD & 1.24 & 1.21 & 1.18 & 1.23 & 1.21 & 1.21 & 19.43 & 19.48 & 19.25 & 3.10 & 2.91 & 2.29 \\
\hline & $\mathrm{CD} 2$ & 1.25 & 1.22 & 1.19 & 1.24 & 1.22 & 1.23 & 19.79 & 19.96 & 19.61 & 3.09 & 2.92 & 2.90 \\
\hline & MS2 & 1.25 & 1.22 & 1.19 & 1.23 & 1.21 & 1.22 & 19.75 & 19.90 & 19.60 & 3.10 & 2.90 & 2.86 \\
\hline & GGCS & 1.26 & 1.22 & 1.19 & 1.23 & 1.21 & 1.22 & 19.65 & 19.92 & 19.57 & 3.09 & 2.89 & 2.86 \\
\hline & GS & 1.36 & 1.29 & 1.27 & 1.04 & 1.02 & 1.04 & 19.16 & 19.29 & 19.14 & 2.58 & 2.35 & 2.35 \\
\hline & GGS & 1.30 & 1.26 & 1.25 & 1.04 & 1.02 & 1.05 & 18.35 & 18.66 & 18.36 & 2.58 & 2.35 & 2.35 \\
\hline
\end{tabular}

differences between the interpolation types under the block matching approach, this means that the patch strategy used in the block matching methodology does not take advantage of finer interpolation schemes and the similarity measures behave in the same fashion as they did without any multi-resolution scheme.

On the other hand, some significant differences are found using the demons approach ( $p$-value $\left.<10^{-2}\right)$, where the linear and cubic schemes show differences with respect to the nearest neighborhood. This was an expected result since the demons transformation model requires interpolations in each iteration. However, no significance difference was found between linear and cubic interpolation and, thus, more complex interpolations do not imply a significant improvement. This result confirms Hypothesis 7, though it shows that the complexity of the interpolation is not a guaranty of better results.

When we analyze together the metrics and the transformation model the importance of joint effects between the similarity measure and the transformation model is confirmed. Note that metrics GS and GGS showed the worse results for motion estimation when no multi-resolution scheme was applied (see Table 4). However, the motion error is reduced due to the more accurate estimations of large deformations while the strain error still remains the best estimated. This improvement is due to a better regularization performed during the multi-resolution scheme, which avoids an excess of regularization for these similarity measures.

\subsection{Effect of myocardial structural information}

The main purpose of a regularization step is to constrain the transformation model to ensure the existence of a solution. Besides, it is possible to introduce myocardial structural information in the transformation by using the normalized convolution to regularize the transformation and improve the ST accuracy. In this section, the Hypothesis 5 is evaluated by studying the influence of the regularization over the ST accuracy:

Experiment 3:. The myocardial structural information is introduced by using the normalized convolution and a myocardial tissue classification as implemented in Curiale et al. (2015).

Results obtained for motion error are shown in Table 6, where we can see a significant improvement of the motion estimation for the demons approach. However, this improvement is not so clear when the block matching method is considered. This fact evidences the importance of the transformation model and the techniques used for interpolating and regularizing the data. The demons philosophy makes use of several regularizations throughout iterations. Thus, considering structural information in each of the regularizations prevent from over regularization and reduces accumulative errors, since the regularization is focusing into the myocardial tissue instead of other non-relevant structures. This observation confirms the results in De Craene et al. (2013), where this effect was already pointed out. The almost negligible effect observed in block matching shows that the performance of this method cannot be easily improved without any multiresolution approach that may take advantage of more regularization steps. We will see this combined effect in the following experiments. The results worsen when the CNR is reduced mainly due to the difficulties of estimating structural information from noisy images.

Regarding the strain estimation, the improvements are consistent with those observed with motion estimation. However, the results observed for low CNR evidence the almost negligible improvement in strain estimation. Thus, in noisy images, the 
Table 6

Global average motion, circumferential strain and radial strain error for the Straus dataset with (Struct. Info.) and without (Simple) myocardial structural information. The relative improvement is shown between parenthesis.

\begin{tabular}{|c|c|c|c|c|c|c|c|c|c|c|c|c|c|}
\hline \multirow[b]{3}{*}{ CNR } & \multirow[b]{3}{*}{ Similarity } & \multicolumn{4}{|c|}{ Average motion error and improvement } & \multicolumn{4}{|c|}{ Average circ. strain error and improvement } & \multicolumn{4}{|c|}{ Average rad. strain error and improvement } \\
\hline & & \multicolumn{2}{|c|}{ Block matching, $\mu[\mathrm{mm}]$ (\%) } & \multicolumn{2}{|c|}{ Demons, $\mu$ [mm] (\%) } & \multicolumn{2}{|c|}{ Block matching, $\mu[\%](\%)$} & \multicolumn{2}{|c|}{ Demons, $\mu$ [\%] (\%) } & \multicolumn{2}{|c|}{ Block matching, $\mu[\%](\%)$} & \multicolumn{2}{|c|}{ Demons, $\mu[\%](\%)$} \\
\hline & & Simple & Struct. Info. & Simple & Struct. Info. & Simple & Struct. Info. & Simple & Struct. Info. & Simple & Struct. Info. & Simple & Struct. Info. \\
\hline \multirow[t]{6}{*}{2.5} & SSD & 3.08 & $3.08(0.2 \%)$ & 0.78 & $0.61(21.4 \%)$ & 19.35 & $19.07(1.4 \%)$ & 2.29 & $2.17(5.6 \%)$ & 23.42 & $23.38(0.2 \%)$ & 20.64 & $16.31(21.0 \%)$ \\
\hline & $\mathrm{CD} 2$ & 3.14 & $3.14(0.2 \%)$ & 0.79 & $0.62(22.4 \%)$ & 19.32 & $19.07(1.2 \%)$ & 2.29 & $2.15(6.0 \%)$ & 23.31 & $23.39(-0.3 \%)$ & 20.86 & $16.38(21.5 \%)$ \\
\hline & MS2 & 3.15 & $3.14(0.2 \%)$ & 0.78 & $0.61(21.1 \%)$ & 19.38 & $19.13(1.3 \%)$ & 2.28 & $2.17(5.2 \%)$ & 23.27 & $23.33(-0.3 \%)$ & 20.25 & $16.21(19.9 \%)$ \\
\hline & GGCS & 3.15 & $3.14(0.2 \%)$ & 0.79 & $0.62(22.0 \%)$ & 19.40 & $19.13(1.4 \%)$ & 2.28 & $2.15(5.6 \%)$ & 23.27 & $23.32(-0.2 \%)$ & 20.43 & $16.28(20.3 \%)$ \\
\hline & GS & 3.08 & $3.07(0.2 \%)$ & 0.93 & $0.68(26.9 \%)$ & 19.06 & $18.88(1.0 \%)$ & 2.15 & $2.04(5.3 \%)$ & 24.56 & $24.68(-0.5 \%)$ & 13.83 & $13.11(5.2 \%)$ \\
\hline & GGS & 3.07 & $3.07(0.2 \%)$ & 0.93 & $0.68(27.0 \%)$ & 18.64 & $18.51(0.7 \%)$ & 2.15 & $2.03(5.3 \%)$ & 24.09 & $24.33(-1.0 \%)$ & 13.64 & $13.04(4.4 \%)$ \\
\hline \multirow[t]{6}{*}{1.2} & SSD & 3.19 & $3.19(0.0 \%)$ & 1.22 & $1.19(3.0 \%)$ & 18.97 & $18.97(0.0 \%)$ & 2.85 & $2.85(-0.1 \%)$ & 25.30 & $25.29(0.0 \%)$ & 30.36 & $30.15(0.7 \%)$ \\
\hline & $\mathrm{CD} 2$ & 3.26 & $3.26(0.0 \%)$ & 1.24 & $1.20(3.2 \%)$ & 19.03 & $19.02(0.1 \%)$ & 2.85 & $2.85(0.0 \%)$ & 24.85 & $24.83(0.1 \%)$ & 30.38 & $30.14(0.8 \%)$ \\
\hline & MS2 & 3.26 & $3.26(0.0 \%)$ & 1.23 & $1.19(3.0 \%)$ & 19.01 & $19.01(-0.0 \%)$ & 2.84 & $2.84(-0.0 \%)$ & 24.82 & $24.83(-0.0 \%)$ & 30.14 & $29.91(0.7 \%)$ \\
\hline & GGCS & 3.26 & $3.26(0.0 \%)$ & 1.24 & $1.20(3.1 \%)$ & 19.02 & $19.01(0.0 \%)$ & 2.84 & $2.84(0.0 \%)$ & 24.83 & $24.84(-0.0 \%)$ & 30.11 & $29.87(0.8 \%)$ \\
\hline & CS & 3.20 & $3.20(0.0 \%)$ & 1.54 & $1.49(3.2 \%)$ & 18.82 & $18.83(-0.0 \%)$ & 3.23 & $3.19(1.2 \%)$ & 25.96 & $25.97(-0.0 \%)$ & 21.14 & $21.30(-0.7 \%)$ \\
\hline & GGS & 3.20 & $3.19(0.0 \%)$ & 1.54 & $1.49(3.1 \%)$ & 18.60 & $18.60(-0.0 \%)$ & 3.23 & $3.19(1.2 \%)$ & 24.99 & $24.97(0.1 \%)$ & 21.03 & $21.19(-0.7 \%)$ \\
\hline
\end{tabular}

Table 7

Average displacement and circumferential strain error, $\mu$, on the Straus dataset for the multi-resolution refinement (MR) and an efficient second-order minimization (ESM). For comparison purpose, it is presented the simplest block matching and demons implementation. The relative improvement is shown between parenthesis.

\begin{tabular}{|c|c|c|c|c|c|c|c|c|c|c|c|}
\hline \multirow[b]{3}{*}{ CNR } & \multirow[b]{3}{*}{ Similarity } & \multicolumn{5}{|c|}{ Average motion error and improvement } & \multicolumn{5}{|c|}{ Average circ. strain error and improvement } \\
\hline & & \multicolumn{2}{|c|}{ Block matching, $\mu[\mathrm{mm}](\%)$} & \multicolumn{3}{|c|}{ Demons, $\mu[\mathrm{mm}](\%)$} & \multicolumn{2}{|c|}{ Block matching, $\mu[\%](\%)$} & \multicolumn{3}{|c|}{ Demons, $\mu[\%](\%)$} \\
\hline & & Simple & MR & Simple & MR & ESM & Simple & MR & Simple & MR & ESM \\
\hline \multirow[t]{6}{*}{2.5} & SSD & 3.08 & $0.96(68.7 \%)$ & 0.78 & $0.74(5.3 \%)$ & $0.48(38.2 \%)$ & 19.35 & $18.95(2.1 \%)$ & 2.29 & $2.29(0.2 \%)$ & $1.90(17.3 \%)$ \\
\hline & $\mathrm{CD} 2$ & 3.14 & $0.97(69.0 \%)$ & 0.79 & $0.75(5.5 \%)$ & $0.49(38.3 \%)$ & 19.32 & $18.95(1.9 \%)$ & 2.29 & $2.29(0.2 \%)$ & $1.89(17.7 \%)$ \\
\hline & MS2 & 3.15 & $0.97(69.0 \%)$ & 0.78 & $0.74(5.2 \%)$ & $0.49(37.0 \%)$ & 19.38 & $18.99(2.0 \%)$ & 2.28 & $2.27(0.4 \%)$ & $1.90(16.9 \%)$ \\
\hline & GGCS & 3.15 & $0.97(69.0 \%)$ & 0.79 & $0.75(5.4 \%)$ & $0.50(37.0 \%)$ & 19.40 & $18.92(2.5 \%)$ & 2.28 & $2.27(0.5 \%)$ & $1.88(17.3 \%)$ \\
\hline & GS & 3.08 & $0.98(68.1 \%)$ & 0.93 & $0.70(25.1 \%)$ & $0.81(12.5 \%)$ & 19.06 & $18.43(3.3 \%)$ & 2.15 & $1.92(10.8 \%)$ & $2.33(-8.5 \%)$ \\
\hline & GGS & 3.07 & $0.99(67.9 \%)$ & 0.93 & $0.70(24.2 \%)$ & $0.81(12.5 \%)$ & 18.64 & $17.72(4.9 \%)$ & 2.15 & $1.92(10.7 \%)$ & $2.33(-8.5 \%)$ \\
\hline \multirow[t]{6}{*}{1.2} & SSD & 3.19 & $1.21(62.0 \%)$ & 1.22 & $1.21(1.2 \%)$ & $0.68(44.7 \%)$ & 18.97 & $19.48(-2.7 \%)$ & 2.85 & $2.91(-2.3 \%)$ & $2.29(19.5 \%)$ \\
\hline & $\mathrm{CD} 2$ & 3.26 & $1.22(62.4 \%)$ & 1.24 & $1.22(1.4 \%)$ & $0.69(44.5 \%)$ & 19.03 & $19.96(-4.9 \%)$ & 2.85 & $2.92(-2.3 \%)$ & $2.28(19.8 \%)$ \\
\hline & MS2 & 3.26 & $1.22(62.4 \%)$ & 1.23 & $1.21(1.6 \%)$ & $0.69(43.8 \%)$ & 19.01 & $19.90(-4.7 \%)$ & 2.84 & $2.90(-1.9 \%)$ & $2.29(19.3 \%)$ \\
\hline & GGCS & 3.26 & $1.22(62.4 \%)$ & 1.24 & $1.21(2.0 \%)$ & $0.70(43.5 \%)$ & 19.02 & $19.92(-4.8 \%)$ & 2.84 & $2.89(-1.9 \%)$ & $2.29(19.3 \%)$ \\
\hline & GS & 3.20 & $1.29(59.7 \%)$ & 1.54 & $1.02(34.0 \%)$ & $1.20(21.8 \%)$ & 18.82 & $19.29(-2.5 \%)$ & 3.23 & $2.35(27.3 \%)$ & $3.30(-2.2 \%)$ \\
\hline & GGS & 3.20 & $1.26(60.6 \%)$ & 1.54 & $1.02(33.7 \%)$ & $1.20(21.9 \%)$ & 18.60 & $18.66(-0.4 \%)$ & 3.23 & $2.35(27.5 \%)$ & $3.30(-2.2 \%)$ \\
\hline
\end{tabular}

structural information is just a technique recommendable when the probabilistic tissue characterization provides good estimates.

\subsection{Effect of multi-resolution and efficient second order minimization strategies}

The tracking in ST is usually carried out by optimizing the transformation model according to a similarity measure. In this section, the influence of the strategy selected for optimization is analyzed by evaluating the Hypotheses 6 and 8 . In particular, we will focus on two popular methodologies: a coarse to fine refinement (multi-resolution) and an efficient second-order minimization (ESM) proposed for demons in Vercauteren et al. (2009). To that aim, the following experiment is proposed:

Experiment 4: The multi-resolution strategy is evaluated for both the block matching and demons approaches using a linear interpolation scheme. On the other hand, the efficient second-order minimization is only evaluated for the diffusion approach.

Table 7 shows the average error for both strategies at two signal to noise levels (CNR $=\{1.2,2.5\})$. The multi-resolution philosophy shows a tremendous improvement for the block matching approach in the motion estimation, obtaining errors below $1 \mathrm{~mm}$. This substantial improvement makes the multi-resolution block matching a technique with similar results than the simple version of demons for motion estimation. However, the strain error is not equally reduced when circumferential strain is considered. This interesting result is due to the great improvement observed in the radial direction, but the not so good improvement in the circumferential direction, where the multi-resolution block matching algorithm still fails.
In general we can conclude that the coarse to fine refinement improves the motion estimation significantly for both methods. However, the strain error slightly improves and it can get even worse in low CNR scenarios. This interesting behavior of demons shows that the iterative optimization of the displacement fields already behaves as the multi-resolution philosophy. There are some interesting exceptions that show a noticeable improvement with those metrics not considering log compression (GS and GGS). This behavior also confirms the importance of the metrics during the regularization steps of demons. Note that the metrics GS and GGS provide a more suitable speckle model which prevents from over regularization. Thus, the multi-resolution scheme leverages this better characterization of speckle to reduce the cumulative error during the multi-resolution approach, resulting in an improvement of both motion and strain estimation.

In conclusion, Hypothesis 6 is discarded in general since the coarse to fine approach improves the motion estimation, though there are some metrics such as GS and GGS where the multiresolution scheme is especially recommended.

Regarding the second-order minimization, Table 7 shows a general improvement in motion estimation and also in strain estimation. However, this minimization scheme is more unstable for metrics whose relation is established as a ratio, which cause worse results in the strain estimation, though the motion error is reduced due to the regularization scheme of demons. Thus, Hypothesis 8 can be also rejected in general, since it is just recommendable when metrics involving difference of intensities (see Table 2).

\subsection{Performance of the complete ST pipeline}

In the previous sections, different parts of a ST method have been independently analyzed. However, the performance of some 
Table 8

Average motion and circumferential strain error, $\mu$, on the Straus dataset for block matching and demons approach with (All) and without (Simple) all the strategies.

\begin{tabular}{|c|c|c|c|c|c|c|c|c|c|}
\hline \multirow[b]{3}{*}{ CNR } & \multirow[b]{3}{*}{ Similarity } & \multicolumn{4}{|c|}{ Average motion error and improvement } & \multicolumn{4}{|c|}{ Average circ, strain error and improvement } \\
\hline & & \multicolumn{2}{|c|}{ Block matching, $\mu[\mathrm{mm}](\%)$} & \multicolumn{2}{|c|}{ Demons, $\mu[\mathrm{mm}](\%)$} & \multicolumn{2}{|c|}{ Block matching, $\mu[\%](\%)$} & \multicolumn{2}{|c|}{ Demons, $\mu[\%](\%)$} \\
\hline & & Simple & All & Simple & All & Simple & All & Simple & All \\
\hline \multirow[t]{6}{*}{2.5} & SSD & 3.08 & $0.97(68.7 \%)$ & 0.78 & $0.48(38.2 \%)$ & 19.35 & $18.85(2.6 \%)$ & 2.29 & $2.07(9.7 \%)$ \\
\hline & $\mathrm{CD} 2$ & 3.14 & $0.98(68.9 \%)$ & 0.79 & $0.49(38.9 \%)$ & 19.32 & $18.92(2.0 \%)$ & 2.29 & $2.06(10.0 \%)$ \\
\hline & MS2 & 3.15 & $0.98(68.9 \%)$ & 0.78 & $0.48(37.8 \%)$ & 19.38 & $18.88(2.6 \%)$ & 2.28 & $2.07(9.5 \%)$ \\
\hline & GGCS & 3.15 & $0.98(68.9 \%)$ & 0.79 & $0.49(38.3 \%)$ & 19.40 & $18.88(2.7 \%)$ & 2.28 & $2.06(9.7 \%)$ \\
\hline & GS & 3.08 & $0.98(68.0 \%)$ & 0.93 & $0.51(45.0 \%)$ & 19.06 & $18.37(3.7 \%)$ & 2.15 & $1.96(8.9 \%)$ \\
\hline & GGS & 3.07 & $0.99(67.8 \%)$ & 0.93 & $0.51(45.0 \%)$ & 18.64 & $17.59(5.6 \%)$ & 2.15 & $2.07(3.6 \%)$ \\
\hline \multirow[t]{6}{*}{1.2} & SSD & 3.19 & $1.21(62.1 \%)$ & 1.22 & $0.57(53.1 \%)$ & 18.97 & $19.46(-2.6 \%)$ & 2.85 & $2.21(22.3 \%)$ \\
\hline & $\mathrm{CD} 2$ & 3.26 & $1.22(62.6 \%)$ & 1.24 & $0.58(53.2 \%)$ & 19.03 & $19.87(-4.4 \%)$ & 2.85 & $2.20(22.8 \%)$ \\
\hline & MS2 & 3.26 & $1.22(62.6 \%)$ & 1.23 & $0.58(52.5 \%)$ & 19.01 & $19.87(-4.6 \%)$ & 2.84 & $2.21(22.2 \%)$ \\
\hline & GGCS & 3.26 & $1.22(62.5 \%)$ & 1.24 & $0.59(52.6 \%)$ & 19.02 & $19.95(-4.9 \%)$ & 2.84 & $2.20(22.6 \%)$ \\
\hline & GS & 3.20 & $1.29(59.8 \%)$ & 1.54 & $1.08(29.6 \%)$ & 18.82 & $19.35(-2.8 \%)$ & 3.23 & $3.16(2.3 \%)$ \\
\hline & GGS & 3.20 & $1.26(60.7 \%)$ & 1.54 & $1.08(30.0 \%)$ & 18.60 & $18.71(-0.6 \%)$ & 3.23 & $3.14(2.9 \%)$ \\
\hline
\end{tabular}

methods are strongly related to other methods along the ST pipeline. The aim of this section is to provide a deeper analysis of the ST accuracy where all the components are combined by means of the following experiment:

Experiment 5. A complete ST pipeline is evaluated for the Strauss data set with the following configuration: (1) using all the similarity measures proposed; (2) both transformation approaches, block matching and demons; (3) the use of myocardial structural information; (4) a multi-resolution approach; and (5) an efficient second-order minimization. Three different contrast values are considered, $\mathrm{CNR}=\{1.2,1.8,2.5\}$.

Results for experiment are collected in Table 8 . We will denote as "All" to the complete pipeline (with structural information, multi-resolution and ESM in the demons transformation) and "Simple" to the simplified pipeline described in Experiment 1. At first glance, these results show a significant improvement in motion estimation when all strategies are applied together. This improvement can go up to $68 \%$ in the case of block matching. However, if factors that are actually contributing to this improvement are carefully checked in Table 7, one can see that the multi-resolution scheme is the key factor whereas the structural information does not play a relevant role for this transformation approach in block matching. This result confirms the behavior already commented in experiment 3, where the importance of regularization steps to reduce cumulative errors was pointed out. Regarding the strain accuracy for block matching, the results shown in Table 7 compared to those of Table 8 evidence no significant improvement with the multi-resolution scheme which shows that structural information does not provide a better refinement than the one obtained from multi-resolution for strain estimation.

On the other hand, the demons approach shows an overall improvement when all the strategies are used. When results obtained for demons in Table 7 and Table 8 are compared, one can see that the motion estimation is not significantly increased in metrics based on difference of intensities. However, those based on ratios (GS and GGS) improve their accuracy to almost the same level as the rest of metrics $(0.51 \mathrm{~mm})$ with a better ST estimate. These results show that the combination of structural information and the ESM technique increases the motion estimation accuracy (from $0.68 \mathrm{~mm}$ and $1.02 \mathrm{~mm}$ respectively to $0.51 \mathrm{~mm}$ ). However, note that the structural information can cause an increase of motion error when the characterization of tissue is poorly estimated in low CNR scenarios (CNR = 1.2), going from $1.49 \mathrm{~mm}$ with structural information (Table 6); $1.02 \mathrm{~mm}$ with multi-resolution (Table 7) to $1.08 \mathrm{~mm}$ with all strategies. This behavior shows that these metrics are more sensitive to structural information and its performance depends on two important steps of the approach: (1) the regularization methodology, which improves with structural information; (2) the optimization scheme, which gets worse when the ESM optimization method is applied. However, the metrics related as difference of intensities show a different behavior: they are sensitive to the structural information (e.g. from $0.78 \mathrm{~m}$ to $0.61 \mathrm{~mm}$, see Table 6, SSD metric) though the efficient second-order optimization performs a better result (e.g. from $0.78 \mathrm{~m}$ to $0.48 \mathrm{~mm}$, see Table 8, SSD metric). The combination of both approaches structural information and ESM provides a similar result in motion estimation, though the strain gets worse due to an excess of regularization due to the normalized convolution (e.g. from $2.29 \%$ circ. strain, to $1.90 \%$ with ESM and $2.07 \%$ with both ESM + structural information, see Table 7 and 8, SSD metric).

Most of the errors measured so far have been given as an average of values along time and space. In what follows we try to have a deeper insight on how the errors are distributed along the cardiac cycle. For the sake of simplicity, only two similarity measures are considered (GGS and GGCS) for the demons approach. Results of the experiments 1 and 5 along the cardiac phase are depicted in Fig. 6. This comparison allows us to see the effect of both metrics throughout the cardiac cycle.

We first focus on the boxplot shown for the higher $\mathrm{CNR}=2.5$, where there is an increment of the mean error and variance along the cardiac phase due to the frame-to-frame error accumulation as expected. Note that the GGCS measure shows a more robust behavior with the simple implementation. The error remains below $1 \mathrm{~mm}$ with a smaller variance than GGS. This result confirms the importance of considering the logarithmic compression into the metric for motion estimation.

When the complete system is considered (structural information + ESM), GGCS still outperforms GGS for motion, but the differences along the cycle have been reduced due to the influence of a better regularization into the ST accuracy mainly due to the good performance of the ESM technique with metrics based on differences of intensities.

From this result one may argue that any similarity measure can be used with similar results, since the complete method improves the motion accuracy to comparable results in all the cases. However, if the complete GGS and the complete GGCS approaches are compared for $\mathrm{CNR}=1.2$ (red line), one can see that the error grows up to $1.5 \mathrm{~mm}$ for GGS, while it is always below $1 \mathrm{~mm}$ for GGCS. This fact is due to the sensitivity of this metric when the ESM is applied. This confirms that the similarity measures also play a role in combination to other configurations. 

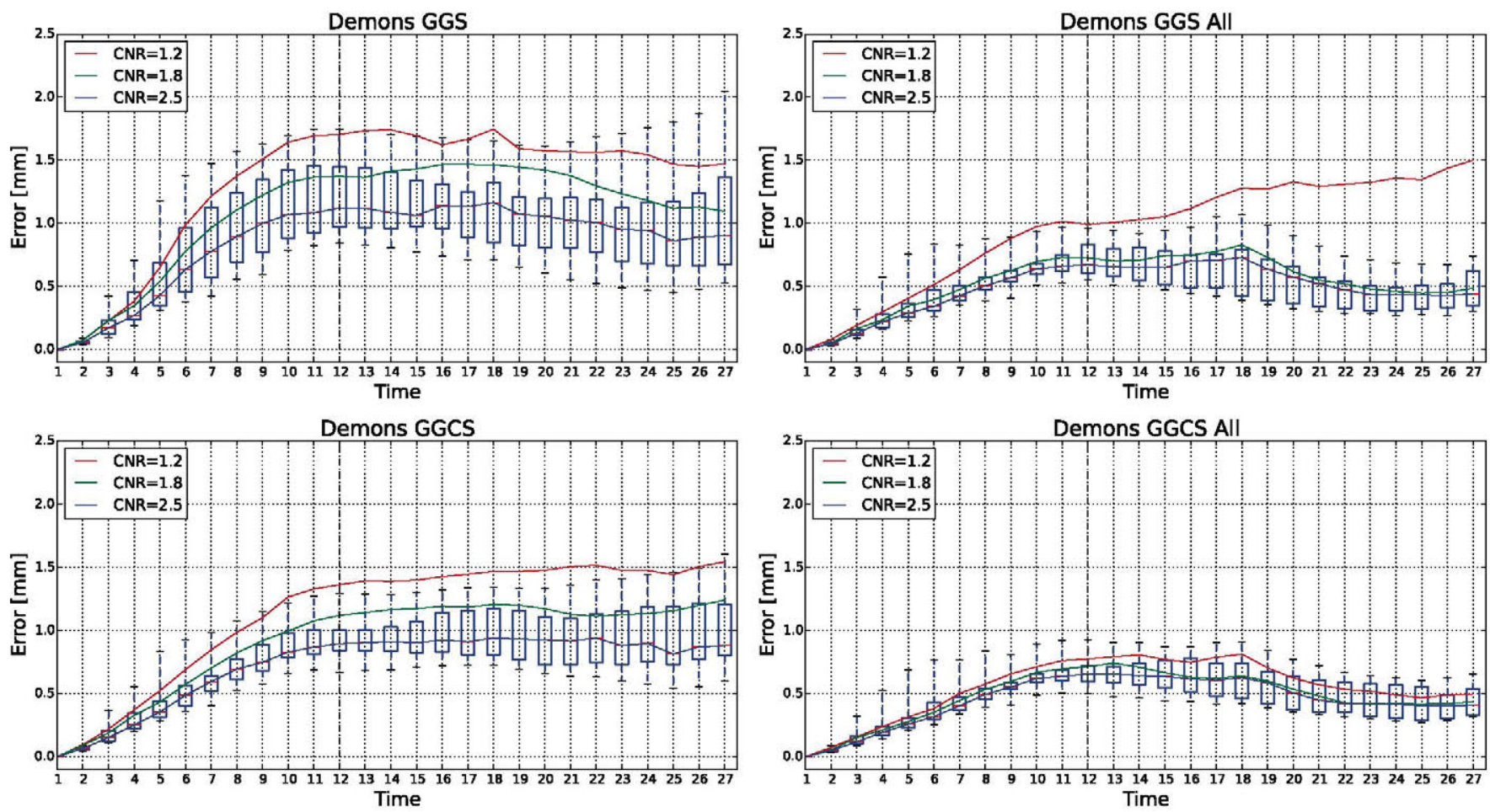

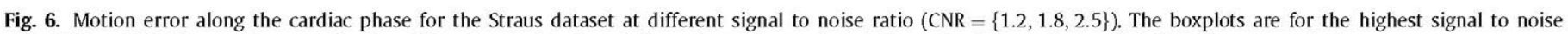
ratio $(\mathrm{CNR}=2.5)$. The median error for the different noise levels and the end-systole are depicted as color lines and a vertical dash line, respectively.

All in all, results show that a complete system usually improves the accuracy of the estimation of motion and strain. However, the different pieces we use to build the complete system must be carefully analyzed. Even when each step improves the accuracy, the final results might be non-optimal. The goodness of each step will strongly depend on the similarity measure used, the transformation method and the optimization technique. What is more, results show that many times the best solution is obtained by only improving one of the steps of the process.

\section{Influence of the pipeline in pathological cases}

In order to get a deeper insight of the relation between the influence of the different strategies into the complete ST pipeline, in this section we study the ST accuracy in scenarios with different pathological cases (four ischemic and two asynchronous) for $\mathrm{CNR}=2.5$. A detailed description about the pathological cases can be found in De Craene et al. (2013).

Fig. 7 shows the global motion error for the ischemic cases and the circumferential strain error for the dyssynchrony cases, where the errors of pathological cases show a similar behavior than those obtained for the normal case. i.e. all the methods have similar motion and strain accuracy with the exception of those derived from the intensity ratio (GS and GGS) which seem to be more sensitive. Therefore, the same conclusions derived for the normal case can be applied for pathological cases. So, in summary, the results obtained for pathological cases do not offer a different analysis than the one already described for normal cases.

\subsection{Discussion on the interdependences}

In the previous sections we have seen that the performance of some methods is strongly related to other methods along the ST pipeline. In order to get a better insight into these relations, in this section we summarize results pointed out in previous sections pointing out their interrelations with other steps.
Table 9 shows the partial contribution of each of the methods to the estimation of motion and strain for the metrics GGCS and GGS in order to see the importance of the relationship between intensities on the optimization techniques applied in other stages of the method. The gain of each of the methods is expressed as a percentage. This table clearly shows how different strategies affect in a different way the final result. Note that, for the similarity measure GGCS, the use of structural information has no effect over the BM approach, while it improves $22 \%$ when using demons as was pointed out in experiment 3 . This behavior was associated to the lack of a proper regularization scheme in the BM approach, which is confirmed due to the improvement up to $69 \%$ obtained when multi-resolution is applied in the BM approach. However, note that the structural information does not provide any further information to the multi-resolution approach since the result obtained applying both together remains the almost the same (68.9 $\%)$. This behavior evidences that the resolution achieved with simple block matching is very reduced to describe the complex deformation of the heart. The multi-resolution scheme overcomes partially this lack of resolution since it achieves a better following of radial deformation, though the circumferential deformation is poorly estimated and causes a poor circumferential strain estimation. A similar conclusion can be obtained for the case of the GGS metric. However, in this case we can observe a reduction of the strain error which is due to the better description of the GGS metric to the statistics of speckle.

In the case of the demons approach for GGCS, the transformation itself improves remarkably the performance of block matching -as expected- due to the regularization inherent to the demons algorithm, which avoids the low resolution problem observed in block matching. Note that the inner regularization intrinsic to the demons approach makes the contribution of a multi-resolution scheme almost negligible (from $0.79 \mathrm{~mm}$ to $0.75 \mathrm{~mm}$ ), while the ESM approach reduces the error to $0.5 \mathrm{~mm}$ reaching a gain of $37 \%$. Besides, though using structural information is more recommendable than using a multi-resolution scheme (improves 22\%), 
Table 9

Improvements for average motion and circumferential strain error for block matching and demons approaches in each of the steps throughout the pipeline for $\mathrm{CNR}=2.5$ and similarity measures GGCS and GGS.

\begin{tabular}{|c|c|c|c|c|c|c|c|c|}
\hline & \multicolumn{4}{|l|}{ GGCS } & \multicolumn{4}{|l|}{ GGS } \\
\hline & \multicolumn{2}{|c|}{ Motion error } & \multicolumn{2}{|c|}{ Circ. strain error } & \multicolumn{2}{|c|}{ Motion error } & \multicolumn{2}{|c|}{ Circ. strain error } \\
\hline & BM & Demons & $\mathrm{BM}$ & Demons & $\mathrm{BM}$ & Demons & $\mathrm{BM}$ & Demons \\
\hline Simple & 3.15 & 0.79 & 19.40 & 2.28 & 3.07 & 0.93 & 18.64 & 2.15 \\
\hline Struct. Info & 3.14 & 0.62 & 19.13 & 2.15 & 3.07 & 0.68 & 18.51 & 2.03 \\
\hline Gain & $0.2 \%$ & $22 \%$ & $1.4 \%$ & $5.6 \%$ & $0.2 \%$ & $27 \%$ & $0.7 \%$ & $5.3 \%$ \\
\hline MR & 0.97 & 0.75 & 18.92 & 2.27 & 0.99 & 0.70 & 17.72 & 1.92 \\
\hline Gain & $69 \%$ & $5.4 \%$ & $2.5 \%$ & $0.5 \%$ & $67.9 \%$ & $24.2 \%$ & $4.9 \%$ & $10.7 \%$ \\
\hline ESM & - & 0.5 & - & 1.88 & - & 0.81 & - & 2.33 \\
\hline Gain & - & $37 \%$ & - & $17.3 \%$ & - & $12.5 \%$ & - & $-8.5 \%$ \\
\hline All & 0.98 & 0.49 & 18.88 & 2.06 & 0.99 & 0.51 & 17.59 & 2.07 \\
\hline Gain & $68.9 \%$ & $38.3 \%$ & $2.7 \%$ & $9.7 \%$ & $67.8 \%$ & $45 \%$ & $5.6 \%$ & $3.6 \%$ \\
\hline
\end{tabular}
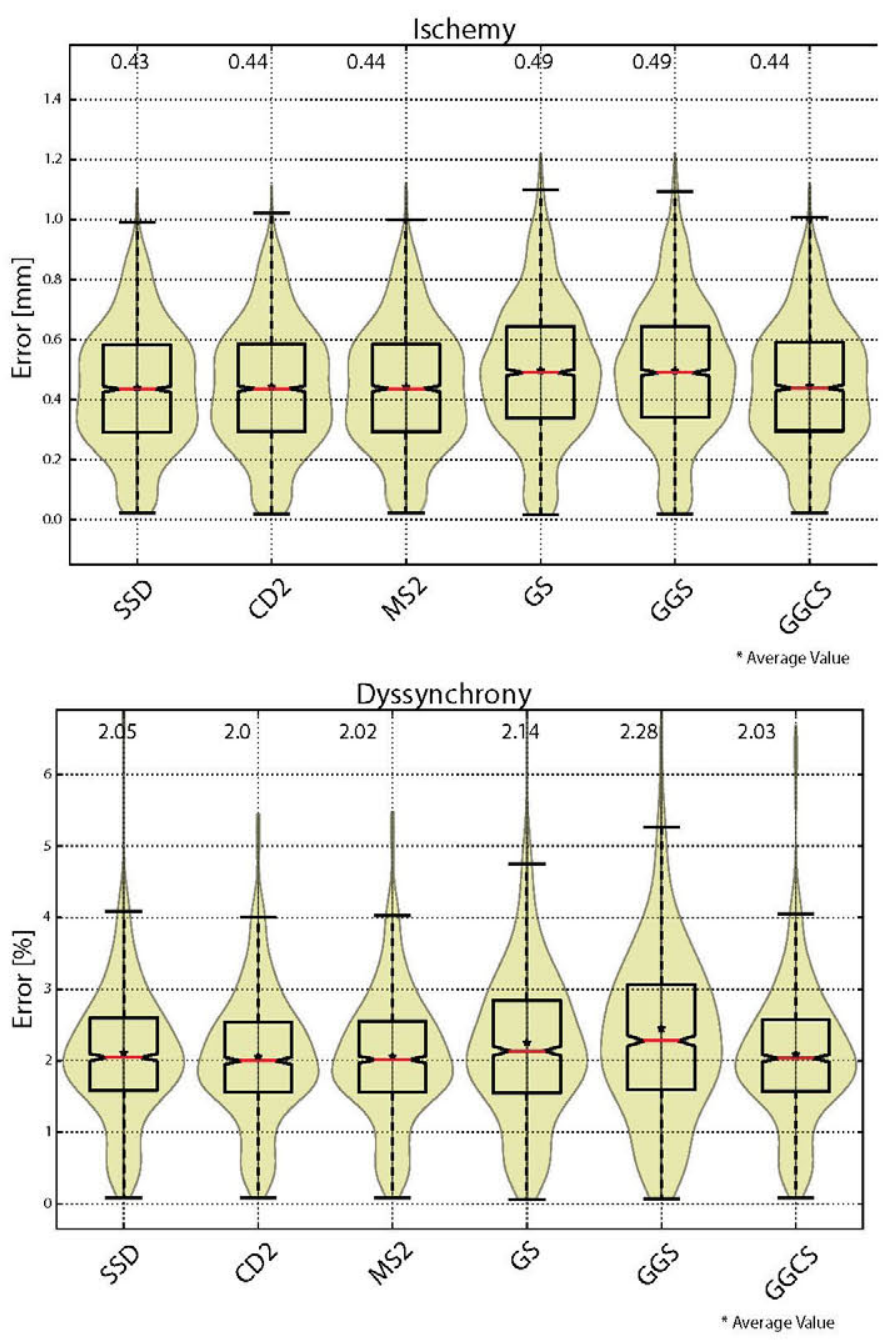

Fig. 7. Global motion and circumferential strain error for the ischemic (top) and dyssynchrony (bottom) cases for the Straus dataset at $C N R=2.5$ respectively.

the EMS is preferable since the reduction obtained from the structural information is already outperformed with the ESM (improves $38.3 \%$ ). However, note that the metric GGS provides worse results with ESM than those with GGCS due to numerical fluctuations due to the ratio of intensities involved in the definition of the metric, thus the structural information contributes better than the ESM approach. The fact that the combination of all techniques reduces the error to similar results obtained from GGCS is due to the combination of the structural information. Note that the numerical fluctuations introduce some errors that affect negatively to the motion estimation. However, the normalized regularization introduced by the structural information in combination of a multiresolution scheme palliates this effect. Actually, this compensatory phenomenon can be better seen for circumferential strain, where the structural information and the multi-resolution scheme affects positively ( $5.3 \%$ and $10.7 \%$ respectively) to the strain estimation but the ESM optimization technique reduces the performance $(-8.5 \%)$, resulting in a complete system with less gain (3.6\%).

\section{Conclusions}

In this paper we studied the influence of the different components of a ST system over motion and strain accuracy in ultrasound images. In this context we studied and identified the relevant relations between the three necessary parts of a ST system: similarity measure, transformation model and optimization strategy. The study was focused on the main hypotheses assumed in the proposals of the state of the art. The conclusions obtained from our analysis confirmed some of the hypotheses but also rejected some them, showing that certain interrelations between similarity measures, transformation models and optimization techniques may offer worse performance than the expected from the contribution of each stage separately. These results reinforce the idea of a whole analysis of a ST system, rather than a part-based analysis.

The study of the influence of similarity measures was performed considering the most relevant measures used in the literature according with an special emphasis on the statistical description of speckle. We distinguished these measures according to the final relationship between intensity levels of the fixed and moving images since it was observed that the performance obtained with different optimization techniques is remarkably affected by this fact. The main conclusions obtained from the analysis of similarity measures are the following:

Conclusion 1. Similarity measures based on a speckle statistical model show more accurate motion and strain estimation. The more detailed the modeling (including spatial and temporal correlation), the more accurate the results. However, the influence of the similarity measures is reduced when the regularization schemes are applied in the transformation model. Generally, these differences may have no practical implications, since they translate in margins lower than $0.3 \mathrm{~mm}$ and $2 \%$ for motion and strain estimation respectively.

Conclusion 2. The improvement of the accuracy due to a transformation model outperforms the improvement due to the similarity measures. Thus, the influence of the transformation model over the 
ST accuracy is higher than the influence of the speckle model itself. This result is consistent with the main conclusion derived from the study proposed in De Craene et al. (2013) where it is observed that most recent ST techniques have relatively similar performances.

Conclusion 3. There is an interdependency between the influence of the similarity measure and the transformation/optimization model. The regularization schemes strongly influence the performance of metrics. Results show that those similarity measures considering the logarithmic compression into the speckle model are more accurate for motion estimation when an iterative optimization approach is used. The logarithmic compression changes the intensity ratio of the speckle model in an intensity difference making the similarity measure more robust to numerical fluctuations. Note that this fact does not mean that the speckle model is more suitable for real US data but for iterative optimization, which increases the effect of regularization for motion estimation, though the performance of strain estimation is reduced due to biases introduced in the regularization.

The analysis of the influence of interpolation on similarity measures based on statistical assumptions was considered by studying three different interpolation schemes (nearest neighbor, linear, cubic) in a multi-resolution implementation in order to increase its contribution. From this analysis the following conclusions were extracted:

Conclusion 4. Although interpolation modifies the statistical model assumed for characterizing the speckle pattern, no reductions of the ST accuracy due to interpolation were observed. On the contrary, linear interpolation in the multi-resolution scheme improves the accuracy of all the metrics and especially those which obtained the best results for strain error. This result not only confirms Hypothesis 3 but also indicates that the way the metrics combine with the transformation model plays an important role.

Conclusion 5. ST accuracy improves when higher order interpolation schemes are used instead of the nearest neighborhood interpolation, which confirms the Hypothesis 7. However, cubic interpolation does not provide any further improvement compared to linear interpolation.

Conclusion 6. The joint effects between the transformation model and the metric are observed with similarity measures based on ratios (without log-compression), which increased their performance in motion estimation due to the refinements of the multiresolution approach, while they provide the best strain estimation.

The inclusion of myocardial structural information by means of normalized convolution was also studied for the different similarity measures and transformation models. The improvement of this technique was shown to be dependent on the transformation philosophy, where iterative transformations ar especially recommended. Thus, the main conclusion is:

Conclusion 7. The use of myocardial structural information in the ST technique significantly improves motion and strain accuracy in iterative approaches like demons due to the iterative error reduction. However, its gain depends on the performance of the probabilistic characterization of tissues.

The analysis of optimization techniques, such as multiresolution and second order minimization, offers very interesting and counterintuitive results:

Conclusion 8. Though the multi-resolution scheme highly contributes in simple approaches such as block matching, other transformation models including regularization steps do not significantly improve their performance with metrics based on difference of intensities. However, metrics based on ratios of intensities improve significantly both the motion and the strain estimation in a multi-resolution approach.

Conclusion 9. Metrics based on ratios of intensities are prone to numerical fluctuations that reduce the performance of strain estimations when the ESM methodology is applied. However, the ESM is especially recommendable with metrics based on difference of intensities.

Finally, the performance of the complete ST pipeline was studied to provide some insights to the interrelations between different combinations of components:

Conclusion 10. Results showed that the block matching approach is greatly improved due to the multi-resolution scheme. Conversely, the inclusion of structural information does not play a relevant role. However, regarding the strain analysis, the overall results evidence that simple transformation models that do not effectively deal with the circumferential estimation of motion (and thus, strain), are not recommended for strain estimation.

Conclusion 11. Similarity measures based on intensity ratios are more sensitive to structural information and its performance depends on the regularization methodology. Thus, multi-resolution is more recommendable than ESM for these metrics.

Conclusion 12. Metrics based on intensity differences improve their performance with the use of the ESM.

Conclusion 13. The use of the myocardial structural information also improves significantly the ST accuracy regardless the similarity measure used.

The results and conclusions obtained throughout this study clearly showed that the election of certain methods along the pipeline must take into account the choices previously made. Methods that independently achieve the best results do not necessarily are optimal when combined together. In fact, the selection of the methodologies to use must not only be based on their individual performance, but on their performance as part of a complete systems where all the parts are interconnected. The selection of a particular step must be based on the choices done for previous and following steps. The goodness of a particular method cannot be guaranteed inside a ST system unless it is tested for the whole pipeline.

The conclusions obtained in this work allow us to provide some recommendations for future research and proposals on ST systems

1. The inclusion of too elaborated similarity measures based on statistics of speckle do not improve significantly the results. The most relevant factor that affects to its performance is the final relationship between intensities, which is a result of considering or not the log-compression in the statistical model.

2. If we are interested just in motion estimation, any similarity measure can be applied with good results, where the most recommended due to its simplicity is the SSD. However, it is important to consider that those metrics based on ratios of intensities should avoid the use of the ESM optimization technique, since it is prone to numerical fluctuations that decrease their performance.

3. If we are interested on strain estimation, metrics without considering log-compression (GS or GGS) are especially recommended when a multi-resolution scheme regularized with structural information by means of normalized convolution is applied. This metrics are more sensitive to the structural information and provide better overall estimation of strain.

4. The regularization step in iterative approaches is of great importance, since its numerical stability and a proper inclusion of 
statistical models can cause unexpected results that may undermine the final results.

5. A linear interpolation scheme is recommended, rather than higher order schemes that do not contribute significantly.

6. The regularization by means of normalized convolution is highly recommended for all similarity measures, especially for those highly sensitive to structural information such as (GS and GGS).

\section{Acknowledgments}

This work was partially supported by the Ministerio de Educación, Ciencia y Tecnología (MECyT), the Consejo Nacional de Investigaciones Científicas y Técnicas (CONICET) and the Universidad Nacional de Cuyo de Argentina. Also it was supported by the Fundación Carolina, the Universidad de Valladolid (FPI-Uva), and the Ministerio de Ciencia e Innovación de España by grant TEC 2013-44194-P. Gonzalo Vegas-Sánchez-Ferrero acknowledges Consejería de Educación, Juventud y Deporte of Comunidad de Madrid and the People Programme (Marie Curie Actions) of the European Union's Seventh Framework Programme (FP7/2007- 2013) for REA grant agreement no. 291820.

\section{References}

Alessandrini, M., Liebgott, H., Barbosa, D., Bernard, O., 2013. Monogenic phase based optical flow computation for myocardial motion analysis in 3d echocardiography. In: Camara, O., Mansi, T., Pop, M., Rhode, K., Sermesant, M., Young, A. (Eds.), Statistical Atlases and Computational Models of the Heart. Imaging and Modelling Challenges. Vol. 7746 of Lecture Notes in Computer Science. Springer, Berlin Heidelberg, pp. 159-168.

Amundsen, B.H., Helle-Valle, T., Edvardsen, T., Torp, H., Crosby, J., Lyseggen, E., et al., 2006. Noninvasive myocardial strain measurement by speckle tracking echocar diography: validation against sonomicrometry and tagged magnetic resonance imaging. J. Am. Coll. Cardiol. 47 (4), 789-793.

Bajcsy, R., Kovačič, S., 1989. Multiresolution elastic matching. Comput. Vision Graph. Image Process. 46 (1), 1-21.

Bargiggia, G.S., Tronconi, L., Sahn, D.J., Recusani, F., Raisaro, A., De Servi, S., Valdes-Cruz, L.M., Montemartini, C., 1991. A new method for quantitation of mitral regurgitation based on color flow Doppler imaging of flow convergence proximal to regurgitant orifice. Circulation 84 (4), 1481-1489.

Beauchemin, S.S., Barron, J.L., 1995. The computation of optical flow. ACM Comput. Surv. 27 (3), 433-466.

Bohs, L., Geiman, B., Anderson, M., Gebhart, S., Trahey, G., 2000. Speckle tracking for multi-dimensional flow estimation. Ultrasonics 38 (1-8), 369-375.

Bohs, L., Trahey, G., 1991. A novel method for angle independent ultrasonic imaging of blood flow and tissue motion. IEEE Trans. Biomed. Eng. 38 (3), 280-286.

Burckhardt, C., 1978. Speckle in ultrasound B-mode scans. IEEE Trans. Sonics Ultra son. 25 (1), 1-6.

Cohen, B., Dinstein, I., 2002. New maximum likelihood motion estimation schemes for noisy ultrasound images. Pattern Recogn. 35 (2), 455-463.

Helle-Valle, T., Crosby, J., Edvardsen, T., Lyseggen, E., Amundsen, B.H., Smith, H.-J., Rosen, B.D., Lima, J.A., Torp, H., Ihlen, H., Smiseth, O.A., 2005. New noninvasive method for assessment of left ventricular rotation. Circulation 112 (20), 3149-3156.

Curiale, A.H., Vegas-Sánchez-Ferrero, G., Aja-Fernández, S., 2013a. Speckle tracking in interpolated echocardiography to estimate heart motion. In: Ourselin, $\mathrm{S}$, Rueckert, D., Smith, N. (Eds.), Functional Imaging and Modeling of the Heart. Vol. 7945 of Lecture Notes in Computer Science. Springer, Berlin Heidelberg, pp. $325-333$.

Curiale, A.H., Vegas-Sanchez-Ferrero, G., Bosch, J.G., Aja-Fernández, S., 2015, A max imum likelihood approach to diffeomorphic speckle tracking for 3D strain esti mation in echocardiography. Med. Image Anal. 24 (1), 90-105.

Curiale, A. H., Vegas-Sanchez-Ferrero, G., Perez-Sanz, T., Aja-Fernandez, S., Strain rate tensor estimation from echocardiography for quantitative assessment of functional mitral regurgitation. In: Biomedical Imaging (ISBI), 2013 IEEE 10th International Symposium on., pp. 788-791.

Dandel, M. Lehmkuhl, H, Knosalla, C, Suramelashvili, N., Hetzer, R, 2009. Strain and strain rate imaging by echocardiography - basic concepts and clinical ap plicability. Current Cardiol. Rev. 5 (2), 133-148.

De Craene, M. Marchesseau, S., Heyde, B., Gao, H., Bernard, O., Alessandrini, M. Piella, G., Porras, A., Saloux, E., Tautz, L., Hennemuth, A., Prakosa, A., Liebgott, H., Somphone, O., Allain, P., Ebeid, S., Delingette, H., Sermesant, M., D’hooge, J., 2013. 3d strain assessment in ultrasound (straus): A synthetic comparison of five tracking methodologies. IEEE Trans. Med. Imag. 32 (9), 1632-1646.

De Craene, M., Piella, G., Camara, O., Duchateau, N., Silva, E., Doltra, A., D'hooge, J., Brugada, J., Sitges, M., Frangi, A.F., 2012. Temporal diffeomorphic free-form deformation: Application to motion and strain estimation from $3 \mathrm{~d}$ echocardiography. Med. Image Anal. 16 (2), 427-450.
Elen, A., Choi, H.F., Loeckx, D., Gao, H., Claus, P., Suetens, P., Maes, F., D’hooge, J., 2008. Three-dimensional cardiac strain estimation using spatio-temporal elastic registration of ultrasound images: A feasibility study. IEEE Trans. Med. Imag. 27 (11), 1580-1591

Friemel, B., Bohs, L., Trahey, G., 1995. Relative performance of two-dimensional speckle-tracking techniques: normalized correlation, non-normalized correlation and sum-absolute-difference. Ultrasonics Symposium, 1995. Proceedings, 1995, Vol. 2.. IEEE, pp. 1481-1484.

Geyer, H., Caracciolo, G., Abe, H., Wilansky, S., Carerj, S., Gentile, F., Nesser, H., Khandheria, B., Narula, J., Sengupta, P., 2010. Assessment of myocardial mechan ics using speckle tracking echocardiography: Fundamentals and clinical applications. J. Am. Soc. Echocardiogr. 23 (4), 351-369.

Helmcke, F., Nanda, N., Hsiung, M., Soto, B., Adey, C., Goyal, R., Gatewood Jr., R. 1987. Color doppler assessment of mitral regurgitation with orthogonal planes. Circulation 75 (1), 175-183.

Heyde, B., Barbosa, D., Claus, P., Maes, F., D'hooge, J., 2013a. Three-dimensional cardiac motion estimation based on non-rigid image registration using a novel transformation model adapted to the heart. In: Camara, O., Mansi, T., Pop, M., Rhode, K., Sermesant, M., Young, A. (Eds.), Statistical Atlases and Computational Models of the Heart. Imaging and Modelling Challenges. Vol. 7746 of Lecture Notes in Computer Science. Springer, Berlin Heidelberg, pp. $142-150$.

Heyde, B., Jasaityte, R., Barbosa, D., Robesyn, V., Bouchez, S., Wouters, P., Maes, F. Claus, P., D'hooge, J., 2013. Elastic image registration versus speckle tracking for 2D myocardial motion estimation: a direct comparison in vivo. IEEE Trans. Med. Imag. 32 (2), 449-459.

Holden, M., 2008. A review of geometric transformations for nonrigid body regis tration. IEEE Trans. Med. Imag. 27 (1), 111-128.

Knutsson, H., Andersson, M., 2005. Morphons: paint on priors and elastic canvas for segmentation and registration. In: Kalviainen, H., Parkkinen, J., Kaarna, A. (Eds.), Image Analysis. Vol. 3540 of Lecture Notes in Computer Science. Springer, Berlin Heidelberg, pp. 292-301.

Knutsson, H., Westin, C., 1993. Normalized and differential convolution: methods for interpolation and filtering of incomplete and uncertain data. Computer Vision and Pattern Recognition, 1993. Proceedings CVPR '93. 1993. IEEE Computer Society Conference on., pp. 515-523.

Konofagou, E., Ophir, J., 1998. A new elastographic method for estimation and imaging of lateral displacements, lateral strains, corrected axial strains and poisson's ratios in tissues. Ultrasound in Med. Biol. 24 (8), 1183-1199.

Leitman, M., Lysyansky, P., Sidenko, S., Shir, V., Peleg, E., Binenbaum, M., Kaluski, E., Krakover, R., Vered, Z., 2004. Two-dimensional strain-a novel software for realtime quantitative echocardiographic assessment of myocardial function. J. Am. Soc. Echocardiogr. 17 (10), 1021-1029.

Lopata, R.G., Nillesen, M.M., Thijssen, J.M., Kapusta, L., de Korte, C.L., 2011. Three-dimensional cardiac strain imaging in healthy children using RF-data. Ultrasound in Med. Biol. 37 (9), 1399-1408.

McDicken, W., Sutherland, G., Moran, C., Gordon, L., 1992. Colour doppler velocity imaging of the myocardium. Ultrasound in Med. Biol. 18 (6-7), 651-654.

Myronenko, A., Song, X., Sahn, D., 2009. Maximum likelihood motion estimation in 3D echocardiography through non-rigid registration in spherical coordinates. In: Ayache, N., Delingette, H., Sermesant, M. (Eds.), Functional Imaging and Modeling of the Heart. Vol. 5528 of Lecture Notes in Computer Science. Springer, Berlin Heidelberg, pp. 427-436.Jun.

Myronenko, A., Song, X., Sahn, D.J., 2007. LV motion tracking from 3D echocardiography using textural and structural information. Medical Image Computing and Computer-Assisted Intervention MICCAI. Vol. 4792 of Lecture Notes in Computer Science. Springer, pp. 428-435.

Nesser, H.-J., Mor-Avi, V., Gorissen, W., Weinert, L., Steringer-Mascherbauer, R., Niel, J., Sugeng, L, Lang, R.M., 2009. Quantification of left ventricular volumes using three-dimensional echocardiographic speckle tracking: comparison with MRI. Eur. Heart J. 30 (13), 1565-1573.

Notomi, Y., Lysyansky, P., Setser, R.M., Shiota, T., Popović, Z.B., Martin-Miklovic, M.G. Weaver, J.A., Oryszak, S.J., Greenberg, N.L., White, R.D., Thomas, J.D., 2005. Measurement of ventricular torsion by two-dimensional ultrasound speckle tracking imaging. J. Am. Coll, Cardiol. 45 (12), 2034-2041.

O'Donnell, M., Skovoroda, A.R., Shapo, B.M., Emelianov, S.Y.S., 1994. Internal displacement and strain imaging using ultrasonic speckle tracking. IEEE Trans. UItrason., Ferroelectr., Freq. Control 41 (3), 314-32.5.

Ommen, S.R., Nishimura, R.A., Appleton, C.P., Miller, F.A., Oh, J.K., Redfield, M.M., Tajik, A.J., 2000. Clinical utility of doppler echocardiography and tissue doppler imaging in the estimation of left ventricular filling pressures: A comparative simultaneous doppler-catheterization study. Circulation 102 (15), $1788-1794$.

Piella, G., Porras, A., De Craene, M., Duchateau, N., Frangi, A., 2013. Temporal diffeomorphic free form deformation to quantify changes induced by left and right bundle branch block and pacing. In: Camara, O., Mansi, T., Pop, M., Rhode, K., Sermesant, M. Young, A. (Eds.), Statistical Atlases and Computational Models of the Heart. Imaging and Modelling Challenges. Vol. 7746 of Lecture Notes in Computer Science, Springer, Berlin Heidelberg, pp. 134-141.

Press, W.H., Teukolsky, S.A., Vetterling, W.T., Flannery, B.P., 2007. Numerical recipes 3rd edition: The art of scientific computing. Cambridge University Press.

Robinson, D., Chen, F., Wilson, L., 1982. Measurement of velocity of propagation from ultrasonic pulse-echo data. Ultrasound in Med. Biol. 8 (4), 413-420.Ultrasonic Mammography 
Somphone, O., Dufour, C., Mory, B., Hilpert, L., Makram-Ebeid, S., Villain, N., Craene, M., Allain, P., Saloux, E., Motion estimation in 3D echocardiography using smooth field registration. In: Camara, O., Mansi, T., Pop, M., Rhode, K., Sermesant, M., Young, A., (eds.) Springer, Berlin Heidelberg. Statistical Atlases and Computational Models of the Heart. Imaging and Modelling Challenges. Lecture Notes in Computer Science, 151-158.

Strintzis, M., Kokkinidis, I., 1997. Maximum likelihood motion estimation in ultrasound image sequences. IEEE Signal Process. Lett. 4 (6), 156-157.

Suffoletto, M.S., Dohi, K., Cannesson, M., Saba, S., Gorcsan, J., 2006. Novel speckle tracking radial strain from routine black-and-white echocardiographic images to quantify dyssynchrony and predict response to cardiac resynchronization therapy. Circulation 113 (7), 960-968.

Tautz, L., Hennemuth, A., Peitgen, H.-O., 2013. Quadrature filter based motion analysis for 3D ultrasound sequences. In: Camara, O., Mansi, T., Pop, M., Rhode, K. Sermesant, M., Young, A. (Eds.), Statistical Atlases and Computational Models of the Heart. Imaging and Modelling Challenges. Vol. 7746 of Lecture Notes in Computer Science, Springer, Berlin Heidelberg, pp. 169-177.

Thirion, J.P., 1998. Image matching as a diffusion process: An analogy with Maxwell's demons. Medical Image Anal. 2 (3), 243-260.

Trahey, G., Allison, J., von Ramm, O., 1987. Angle independent ultrasonic detection of blood flow. IEEE Trans. Biomed. Eng. BME-34 (12) 965-967.

Trahey, G., Hubbard, S., von Ramm, O., 1988. Angle independent ultrasonic blood flow detection by frame-to-frame correlation of B-mode images. Ultrasonics 26 (5), 271-276.

Trahey, G., Smith, S., von Ramm, O., 1986. Speckle pattern correlation with lateral aperture translation: Experimental results and implications for spatial compounding. IEEE Trans. Ultrason. Ferroelectr. Freq. Control 33 (3), 257-264.
Vegas-Sanchez-Ferrero, G., Aja-Fernandez, S., Palencia, C., Martin-Fernandez, M. 2012. A generalized Gamma mixture model for ultrasonic tissue characterization. Comput. Math. Methods Med, 2012, 1-25.(Article ID 481923)

Vegas-Sanchez-Ferrero, G., Seabra, J., Rodriguez-Leor, O., Serrano-Vida, A., Aja-Fernandez, S., Palencia, C., Martin-Fernandez, M., Sanches, J., 2014. Gamma mixture classifier for plaque detection in intravascular ultrasonic images, IEEE Trans, UItrason., Ferroelectr., Freq. Control 61 (1), 44-61.

Vercauteren, T., Pennec, X., Perchant, A., Ayache, N., 2009. Diffeomorphic demons: Efficient non-parametric image registration. Neurolmage 45 (1, Supplement 1) S61-S72.

Voigt, J.-U., Exner, B., Schmiedehausen, K., Huchzermeyer, C., Reulbach, U., Nix dorff, U., Platsch, G., Kuwert, T., Daniel, W.G., Flachskampf, F.A., 2003. Strain-rate imaging during dobutamine stress echocardiography provides objective evidence of inducible ischemia. Circulation 107 (16), 2120-2126.

van Wijk, M., Thijssen, J., 2002. Performance testing of medical ultrasound equipment: fundamental vs. harmonic mode. Ultrasonics 40 (1-8), 585-591.

Yeung, F., Levinson, S., Fu, D., Parker, K., 1998, Feature-adaptive motion tracking of ultrasound image sequences using a deformable mesh. IEEE Trans. Med. Imag. 17 (6), 945-956.

Yeung, F., Levinson, S.F., Parker, K.J., 1998. Multilevel and motion model-based ultrasonic speckle tracking algorithms. Ultrasound in Med. Biol. 24 (3), 427-441.

Yue, Y., Clark, J., Khoury, D., 2009. Speckle tracking in intracardiac echocardiography for the assessment of myocardial deformation. IEEE Trans. Biomed. Eng. 56 (2) $416-425$. 